2010-04-19

\title{
Development of Criteria for Lamina Emergent Mechanism Flexures with Specific Application to Metals
}

Devin Bradley Ferrell

Brigham Young University - Provo

Follow this and additional works at: https://scholarsarchive.byu.edu/etd

Part of the Mechanical Engineering Commons

\section{BYU ScholarsArchive Citation}

Ferrell, Devin Bradley, "Development of Criteria for Lamina Emergent Mechanism Flexures with Specific Application to Metals" (2010). Theses and Dissertations. 2109.

https://scholarsarchive.byu.edu/etd/2109

This Thesis is brought to you for free and open access by BYU ScholarsArchive. It has been accepted for inclusion in Theses and Dissertations by an authorized administrator of BYU ScholarsArchive. For more information, please contact scholarsarchive@byu.edu, ellen_amatangelo@byu.edu. 


\title{
DEVELOPMENT OF CRITERIA FOR LAMINA EMERGENT \\ MECHANISM FLEXURES WITH SPECIFIC \\ APPLICATION TO METALS
}

\author{
Devin B. Ferrell \\ A thesis submitted to the faculty of \\ Brigham Young University \\ in partial fulfillment of the requirements for the degree of \\ Master of Science
}

Spencer P. Magleby, Chair

Larry L. Howell

Robert H. Todd

Department of Mechanical Engineering

Brigham Young University

August 2010

Copyright (C) 2010 Devin B. Ferrell

All Rights Reserved 



\author{
ABSTRACT \\ DEVELOPMENT OF CRITERIA FOR LAMINA EMERGENT \\ MECHANISM FLEXURES WITH SPECIFIC \\ APPLICATION TO METALS \\ Devin B. Ferrell \\ Department of Mechanical Engineering \\ Master of Science
}

This thesis introduces new revolute and torsional lamina emergent mechanism (LEM) flexure designs that are suited for use in metals. Previous LEM flexures have been designed for use in highly elastic materials, such as polymers. In extending LEM flexure designs to metals, a LEM flexure design criteria is also introduced. The LEM flexure criteria is based on relative performance between the LEM flexure and a performance datum which the LEM flexure must improve upon. This performance datum, or benchmark, is a section of lamina that is of the same overall length, width, and thickness as the LEM flexure. An analysis of the revolute and torsional metal LEM flexures, based on the LEM flexure criteria, is performed and both are found to successfully meet the criteria. A brief comparative performance study is also carried out between a basic crank-slider mechanism to which the revolute and torsional metal LEM flexures have and have not been applied. The revolute and torsional metal LEM flexures are found to improve the crank-slider performance.

Keywords: compliant mechanisms, laminate emergent mechanisms, flexure criteria, sheet metals 



\section{ACKNOWLEDGMENTS}

I would like to acknowledge and thank the United States National Science Foundation for providing the necessary support and funding for this work (through grant CMMI-0800606).

I would also like to thank my graduate advisor, Dr. Spencer Magleby, for his invaluable guidance, input, direction, and counsel that enabled me to successfully complete each step along the way to the ultimate completion of this work. Also, I would like to thank Dr. Larry Howell and Dr. Robert Todd for the time they took to review my work and provide their much appreciated feedback and counsel.

There are many students in the Brigham Young University Compliant Mechanisms Research lab that deserve my utmost thanks. Specifically, I would like thank Amanda Pruett for constructing and programing the force-deflection tester, Yanal Isaac for providing his expert skill in the manufacture and testing of prototypes, as well as Nathan Llewellyn for his role in the completion of the RUFF and TUFF crank-slider prototype.

I owe special gratitude to my parents, who instilled in me early in life the value of hard work, personal integrity, and what it means to have goals that you are willing to "walk over hot coals" to accomplish.

Additionally, I owe tremendous thanks to my sweet wife, Eloise, for her never failing love, encouragement, and support throughout the course of my schooling.

Most of all, I would like to thank my Heavenly Father, and his son Jesus Christ, who have been the progenitors of every accomplishment, success, and blessing that I have attained in my life, including the successful completion of this work. 



\section{TABLE OF CONTENTS}

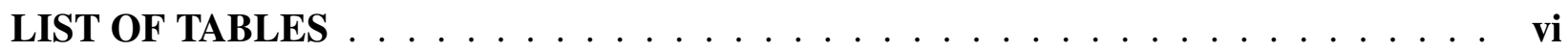

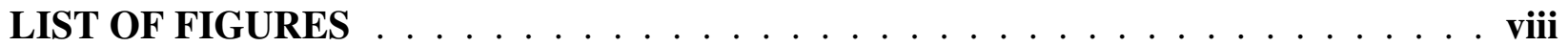

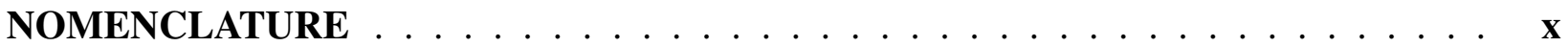

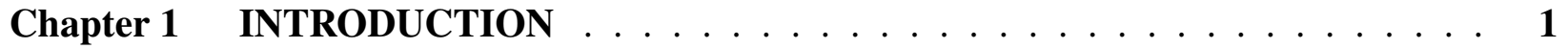

1.1 Lamina Emergent Mechanisms (LEMs) . . . . . . . . . . . . . . . 1

1.2 LEM Flexures . . . . . . . . . . . . . . . . . . . . . . . 2

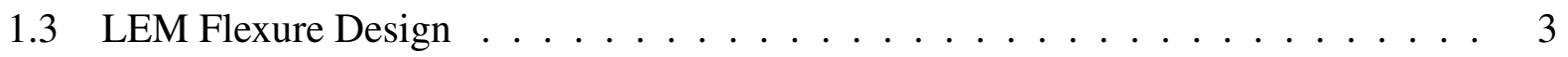

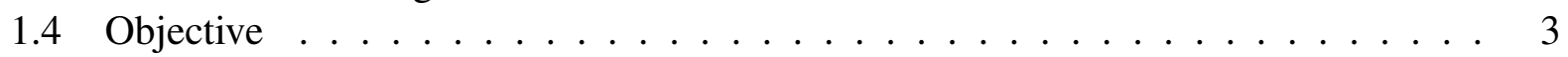

Chapter 2 LEM FLEXURE CRITERIA . . . . . . . . . . . . . . . . . . 5

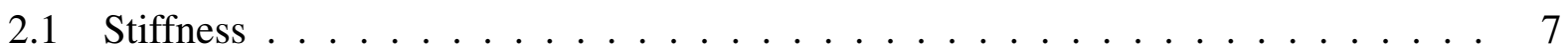

2.2 Yield Strength . . . . . . . . . . . . . . . . . . . . . . . . . . . . . . . . . .

2.3 Off-axis Stiffness Ratio . . . . . . . . . . . . . . . . . . . 9

Chapter 3 METAL LEM FLEXURES . . . . . . . . . . . . . . . . . . . . 11

3.1 Forming LEM Flexures from Metals . . . . . . . . . . . . . . . . . . . . 11

3.2 Other Advantages of Metal LEMs . . . . . . . . . . . . . . . . . 12

Chapter 4 METAL LEM FLEXURE FOR BENDING: REVOLUTE U-FORM FLEXURE (RUFF) . . . . . . . . . . . . . . . . . . . . . . . . . . 13

4.1 RUFF Analysis . . . . . . . . . . . . . . . . . . . . 14

4.1.1 RUFF Closed-form Solution . . . . . . . . . . . . . . . . 16

4.1 .2 RUFF FEA Model . . . . . . . . . . . . . . . . . . . 18

4.1.3 RUFF Prototype Manufacture and Testing . . . . . . . . . . . . . . . . . . 19

4.2 RUFF Versus Benchmark: Force-deflection . . . . . . . . . . . . . . . . 20

4.3 RUFF Versus Benchmark: Off-axis Stiffness Ratio . . . . . . . . . . . . . . 21

4.4 RUFF Conclusion . . . . . . . . . . . . . . . . . . . . . 24

Chapter 5 METAL LEM FLEXURE FOR TORSION: TORSIONAL U-FORM FLEXURE (TUFF) . . . . . . . . . . . . . . . . . . . . . . . 25

5.1 TUFF Analysis . . . . . . . . . . . . . . . . . 26

5.1 .1 TUFF FEA Model . . . . . . . . . . . . . . . . 27

5.1 .2 TUFF Prototype Manufacture and Testing . . . . . . . . . . . . . . 27

5.2 TUFF Versus Benchmark: Torque-rotation . . . . . . . . . . . . . . . . 28

5.3 TUFF Versus Benchmark: Off-axis Stiffness Ratio . . . . . . . . . . . . . 31

5.4 TUFF Conclusion . . . . . . . . . . . . . . . . 33

Chapter 6 APPLICATION OF METAL LEM FLEXURES . . . . . . . . . . . . . 35

6.1 Compound LET Joint . . . . . . . . . . . . . . . . . . . . . . . . 35 
6.2 RUFF and TUFF Crank-slider . . . . . . . . . . . . . . . . . . 35

6.3 RUFF and TUFF Crank-slider FEA and Prototype . . . . . . . . . . . . . . . . 37

6.4 RUFF and TUFF Crank-slider FEA Results and Discussion of Results . . . . . . . 37

6.5 Application of Metal LEM Flexures Conclusion . . . . . . . . . . . . . . . . . . 39

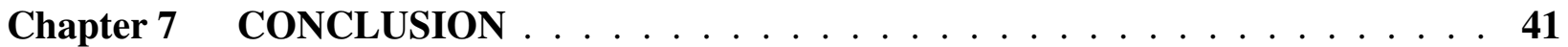

REFERENCES ............................ 43 


\section{LIST OF TABLES}

4.1 RUFF prototype dimensions $\ldots \ldots \ldots \ldots$

5.1 TUFF prototype dimensions $\ldots \ldots \ldots \ldots$ 


\section{LIST OF FIGURES}

1.1 Ortho-planar compliant spring [1] shown in both undeflected (top) and deflected (bottom) positions: example of a fully-compliant mechanism designed to use only flexible members to provide the desired deflection. . . . . . . . . . . . . . . 2

1.2 Example of a four-bar lamina emergent mechanism (with Lamina Emergent Tor-

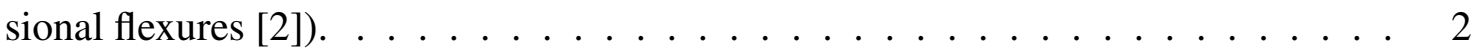

1.3 The LEM flexure (below) is the same original length, $L$, width, $b$, and thickness, $h$, as the performance datum geometry, or benchmark (top). . . . . . . . . . . 4

2.1 Example of a (a) LEM member providing distributed out-of-plane motion under bending load and (b) a LEM flexure providing localized out-of-plane motion under the same bending load as in (a). . . . . . . . . . . . . . . . . .

2.2 Example of a progression (depicted by arrows) from a (a) lamina to a (b) benchmark and the creation of a (c) LEM flexure created in the benchmark through forming, (d) LEM flexure in the benchmark through removal of material, or otherwise manipulation of the lamina without the use of forming processes, (e) flexure created in the benchmark through both removal of material and forming. . . . . . 7

2.3 Optional caption for list of figures . . . . . . . . . . . . . . 8

2.4 A door hinge is an example where off-axis stiffness in rotation about the $x$ or $y$ axes is theoretically infinite. . . . . . . . . . . . . 9

3.1 Examples of how sheet metal forming processes can be used to (a) decrease width, (b) decrease thickness and (c) increase length to form a LEM flexure. . . . . . . . . 11

3.2 Examples of how sheet metal forming process can be used to increase the crosssection moment of inertia through changing the cross-sectional profile of the benchmark. . . . . . . . . . . . . . . . . . . . . . 12

4.1 Revolute U-Form Flexure (RUFF) in (a) deflected and (d) undeflected positions. . . 13

4.2 Schematic of post-formed RUFF. . . . . . . . . . . . . . . . . . . . . . . 14

4.3 Schematic of RUFF fabrication set-up. . . . . . . . . . . . . . . . . . 15

4.4 Both the undeflected and deflected positions of the full RUFF closed-form model. . 16

4.5 An FEA of rendering of the RUFF in both deflected and undeflected positions. . . . 18

4.6 Schematic of force-deflection tester used to determine the stiffness characteristics of the RUFF prototype. . . . . . . . . . . . . . . . . . . . . . . . . 19

4.7 Photographs of (a) the RUFF prototype and (d) the RUFF prototype deflected in the force-deflection tester. . . . . . . . . . . . . . . . . . . 20

4.8 Force-deflection plot of the RUFF closed-form solution, FEA simulation, and prototype test data along with a force-deflection plot of the benchmark. The vertical dotted-lines emphasize the point at which the maximum equivalent stress $\left(\sigma_{\max }\right)$ has exceeded the material yield stress $\left(\sigma_{\text {yield }}\right) \ldots \ldots \ldots \ldots \ldots \ldots \ldots$

4.9 Force-deflection plot of non-dimensionalized RUFF data. . . . . . . . . . . . . . 22

4.10 Representation of desired (moment, $M_{z}$ ) and undesired (torsion, $T_{x}$ ) loading shown on both the (a) benchmark and the (b) RUFF. . . . . . . . . . . . . . . 22

4.11 Comparison of off-axis stiffness ratio between the RUFF and benchmark. . . . . . 23 
5.1 Torsional U-Form Torsional (TUFF) in (a) deflected and (d) undeflected positions. . 25

5.2 FEA deformation plot of TUFF rotated 45 degrees under end torque. . . . . . . . 26

5.3 FEA equivalent stress plot of TUFF displaced through $45^{\circ}$ of rotation. . . . . . . . 27

5.4 Schematic of torque-rotation tester set-up used to determine the stiffness characteristics of the TUFF prototype. . . . . . . . . . . . . . . . . . 28

5.5 Optional caption for list of figures . . . . . . . . . . . . . . . . . . . 29

5.6 Torque-rotation plot of the TUFF FEA simulation and prototype test data along with a torque-rotation plot of the benchmark. The vertical dotted-lines emphasize the point at which the maximum stress $\left(\sigma_{\max }\right)$ in the LEM profile or TUFF has reached the material yield stress $\left(\sigma_{\text {yield }}\right) \ldots \ldots \ldots \ldots$. . . . . . . . . 29

5.7 Plots of: The change in the ratio of the maximum rotation of the TUFF, $\theta_{T U F F}$, over the maximum rotation of the benchmark, $\theta_{\text {benchmark }}$, as (a) the ratio of lengthover- width is varied with the thickness held constant and as (b) thickness is varied and the length-over-width ratio is held constant. As well as the change in the ratio of the stiffness of the TUFF, $k_{T U F F}$, over the stiffness of the benchmark, $k_{\text {benchmark }}$, as (c) the ratio of length-over- width is varied with the thickness held constant and as (d) thickness is varied and the length-over-width ratio is held constant. . . . . . 30

5.8 Representation of undesired $\left(M_{z}\right)$ and desired $\left(T_{x}\right)$ loading shown on both the (a) benchmark and the (b) TUFF. . . . . . . . . . . . . . . . . 32

5.9 Off-axis stiffness comparison of the TUFF and benchmark. . . . . . . . . . . . 32

6.1 LET joint [2](top) compared to the compound LET joint (bottom), which replaces the LET members in torsion with TUFFs and the LET members in bending with RUFFs. . . . . . . . . . . . . . . . . . . . . . . . . . . . . . . .

6.2 Schematic of the LEM crank-slider design. . . . . . . . . . . . . . . . . 36

6.3 Renderings of the unactuated (a) and actuated (b) FEA model of the RUFF and TUFF crank-slider, as well as photographs of the RUFF and TUFF crank-slider prototype in both the (c) unactuated and (d) actuated positions. . . . . . . . . . . 38

6.4 Comparision plot of the stress behavior for both the LEM crank-slider with standard LET joints and the LEM crank-slider with compound RUFF and TUFF LET joints as $\varphi$ is increased. . . . . . . . . . . . . . . . . . 39 


\section{NOMENCLATURE}

\begin{tabular}{|c|c|}
\hline$h$ & Thickness of lamina \\
\hline$b$ & Overall width of LEM flexure and Benchmark \\
\hline$L$ & Overall length of LEM flexure and Benchmark \\
\hline$F$ & Applied Force \\
\hline$M$ & Applied Moment \\
\hline$I$ & Moment of interia \\
\hline$K$ & Polar moment of interia for rectangular cross-sections \\
\hline$E$ & Young's modulus \\
\hline$G$ & Shear modulus \\
\hline$\delta$ & Linear deflection \\
\hline$\theta$ & Rotational deflection \\
\hline$h_{f}$ & Post-formed thickness \\
\hline$l$ & In reference to the RUFF: Length of stright section \\
\hline & $\begin{array}{l}\text { In reference to the TUFF: Length of section where the cross-sectional profile transitions } \\
\text { from u-form to flat }\end{array}$ \\
\hline & $\begin{array}{l}\text { In reference to the RUFF and TUFF crank-slider: Length of RUFF and TUFF crank-slider } \\
\text { members }\end{array}$ \\
\hline$b_{s}$ & RUFF slot width \\
\hline$n$ & Number of slots within the RUFF \\
\hline$b_{f}$ & Width of formed sections between slots within the RUFF \\
\hline$y_{i}$ & Linear deflection in direction $i$ \\
\hline$F_{i}$ & Applied force in direction $i$ \\
\hline$U$ & Total strain enegry within an elastic system \\
\hline$I_{S}$ & Cross-sectional moment of interia for straight section of the RUFF \\
\hline$I_{c}$ & Cross-sectional moment of interia for curved section of the RUFF \\
\hline$\sigma_{\max }$ & Maximum stress \\
\hline$\sigma_{\text {yield }}$ & Yield stress \\
\hline$\alpha^{2}$ & Non-dimensionalize force \\
\hline$l_{s}$ & TUFF slot width \\
\hline$l_{f}$ & Width of formed sections between TUFF slots \\
\hline$e$ & Distance between the outside edge of TUFF and outside edge of TUFF slots \\
\hline$k$ & Stiffness \\
\hline . & RUFF and TUFF crank-slider degree of rotation \\
\hline
\end{tabular}




\section{CHAPTER 1. INTRODUCTION}

Compliant mechanisms achieve all or some of their motion from the deflection of both flexible members and joints [1-3]. In comparison, rigid-body mechanisms achieve all of their motion through ridig-body joints (i.e. revolute joints, sliders, cams) [4]. Compliant mechanisms have several potential advantages over rigid-body mechanisms. Among others, they can be lighter, lower cost, more precise, more reliable, and require fewer parts. Fully compliant mechanisms are a type of compliant mechanism that receive all of their motion from the deflection of flexible members and joints (an example of a fully compliant mechanism that uses only flexible members to achive its motion is shown in Figure 1.1).

\subsection{Lamina Emergent Mechanisms (LEMs)}

Lamina emergent mechanisms are mechanical devices fabricated from planar materials (lamina) with motion that emerges out of the fabrication plane [5,6] (see Figure 1.2). LEMs have the same advantages as other compliant mechanisms and can be fully manufactured through inexpensive industrial processes (i.e. shearing, punching, blanking, etc.). The planar nature of LEMs also provides advantages in shipping and storage costs [6].

Past research in LEMs has generated LEM designs that provide good mechanical behavioral characteristics when made from polymers or paper-based materials, but provide less motion when when made from metals $[2,7]$. This research advances LEM design from metals.

The unique mechanical characteristics of metals provide several advantages over other materials in various applications. Some advantages are: good performance in high-temperature environments, low susceptibility to creep, and a more predicable fatigue life [8]. Metal LEMs can provide novel solutions in heat transfer, manufacturing, and design for assembly problems. 

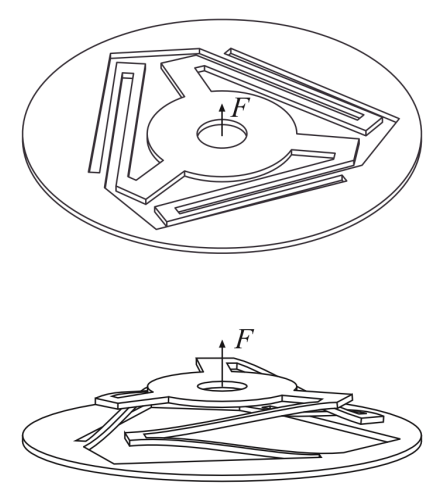

Figure 1.1: Ortho-planar compliant spring [1] shown in both undeflected (top) and deflected (bottom) positions: example of a fully-compliant mechanism designed to use only flexible members to provide the desired deflection.

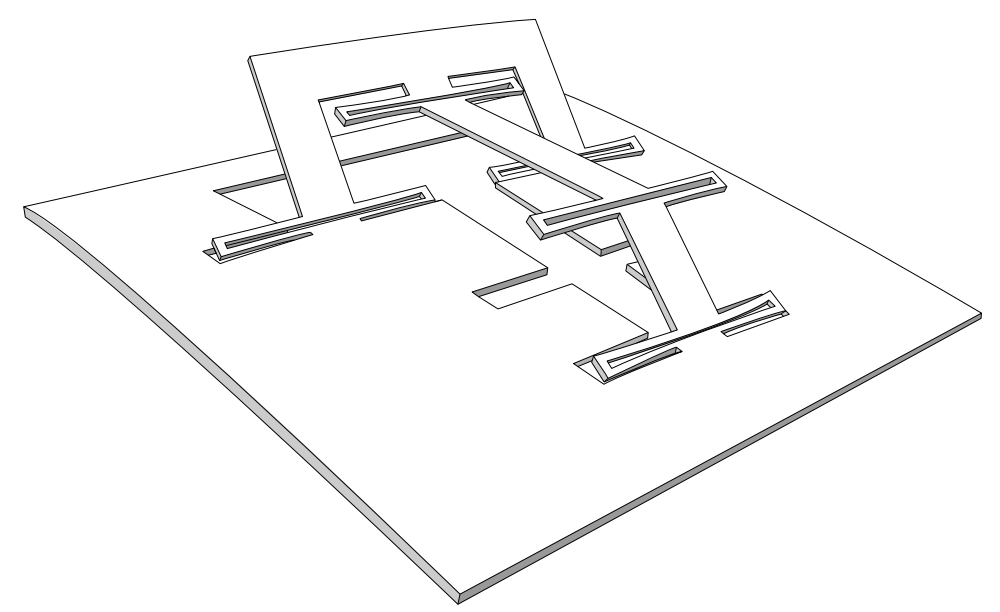

Figure 1.2: Example of a four-bar lamina emergent mechanism (with Lamina Emergent Torsional flexures [2]).

\subsection{LEM Flexures}

Many LEMs achieve their motion through the deflection of long flexible members [1]. While long, flexible members may provide the desired range of motion, they typically have poor off-axis stiffness and have a much larger footprint that a rigid-body counterpart with an equivalent range of motion (i.e. two rigid members coupled by a pin joint) [9]. Also, the exclusive use of long flexible members in LEMs limits the potential mechanism complexity because only one type of deflection is available to achieve the desired motion [10]. This potential limitation in mechanism complexity may also exclude the ability to adapt to LEMs optimization methods that have been developed for non-planar compliant mechanisms [11, 12]. 
The disadvantages of long flexible members represent the need to design LEM specific compliant joints that are analogous to rigid-body joints; i.e. revolute, prismatic or universal joints [13]. The Lamina Emergent Torsional (LET) joint [2] shown in Figure 1.2 is an example of a LEM specific joint compliant revolute joint. In order to eliminate any confusion between LEM joints, used for providing localized motion relative to LEM members, and joints used to rigidly join two parts together (i.e. butt joints) LEM joints will henceforth be referred to as LEM flexures.

\subsection{LEM Flexure Design}

In advancing LEM design to include metals it is advantageous that a performance criteria be created for the design and evaluation of LEM flexures [13]. For such a performance criteria to be realized, identification must be made of the base-line performance, or performance datum, from which to compare a LEM flexure's performance. The performance datum will be a LEM member that is of the same original length, thickness, and width of a proposed LEM flexure design, prior to its manufacture (see Figure 1.3). To simplify the following discussion, the performance datum geometry will be referred to as the benchmark. Therefore, the performance criteria, or LEM flexure criteria, will be a tool by which a designer can evaluate the relative performance of a proposed LEM flexure with respect to its benchmark. If a proposed LEM flexure does not produce improved performance over the benchmark, according to the LEM flexure criteria, then it cannot be considered as a viable LEM.

\subsection{Objective}

The objective of this thesis is threefold. The first is to provide a LEM flexure criteria for use in comparing the performance of proposed LEM flexure designs to the performance of their associated benchmarks. The second is to introduce two new LEM flexure designs specific to metals that meet the LEM flexure criteria provided in the first objective. The first of these designs, the Revolute U-Form Flexure (RUFF), is specifically designed for bending applications. The other, the Torsional U-Form Flexure (TUFF), is designed for torsional applications. Each of these flexures are similarly analyzed and tested to check the LEM flexure performance against each corresponding benchmark, and also to provide the designer a basic understanding of the mechanical 


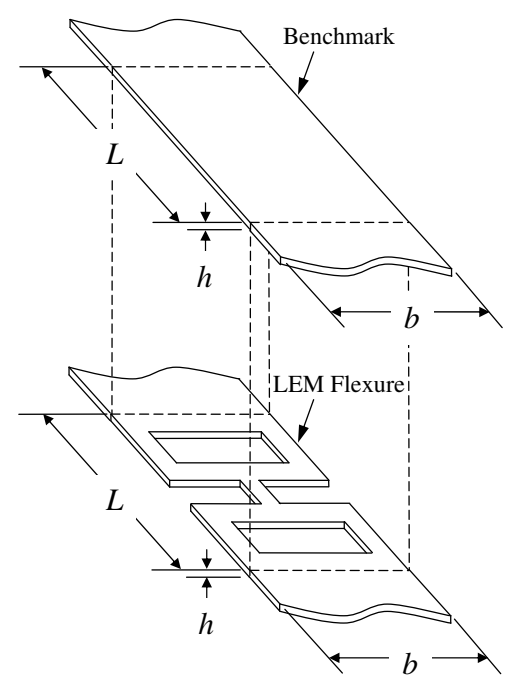

Figure 1.3: The LEM flexure (below) is the same original length, $L$, width, $b$, and thickness, $h$, as the performance datum geometry, or benchmark (top).

characteristics of each LEM flexure design. The third objective is to provide an analysis the RUFF and TUFF in a LEM application. 


\section{CHAPTER 2. LEM FLEXURE CRITERIA}

A LEM flexure can be conceptually defined as different from a LEM member because it provides a localized motion. A LEM member provides a distributed motion, where the desired motion is carried out across the length of the member (see Figure 2.1(a)). A LEM flexure focuses and localizes motion at a specific location (see Figure 2.1(b)) $[14,15]$. To provide this localized motion, a LEM flexure must possess differentiating qualities that create desirable performance over the benchmark (see Figure 2.2(b)). This relationship between the LEM flexure and benchmark is best understood if analyzed in terms of relative performance. How much less stiff the LEM flexure is in relation to its initial LEM member geometry is an example of a performance comparison.

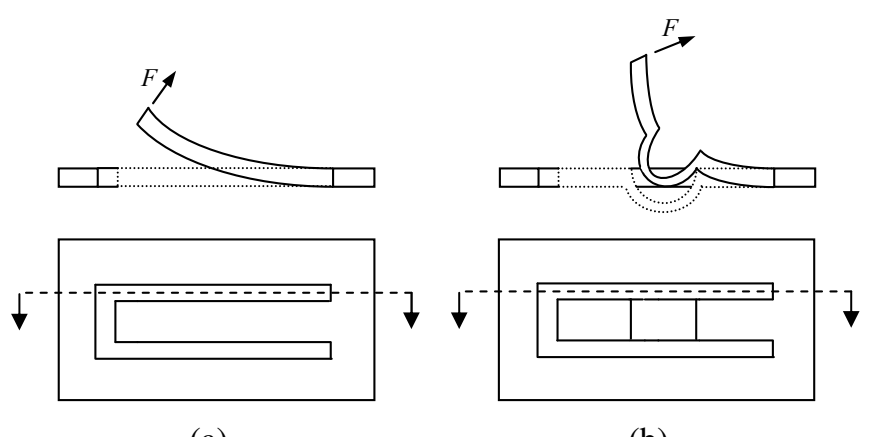

(a)

(b)

Figure 2.1: Example of a (a) LEM member providing distributed out-of-plane motion under bending load and (b) a LEM flexure providing localized out-of-plane motion under the same bending load as in (a).

To further qualify this relative performance, and provide the means by which a LEM flexure can be qualified as a flexure, it is proposed that a relative LEM-flexure-to-benchmark performance criteria be established. In establishing such a criteria it is important to consider what qualities should be improved by a proposed LEM flexure design. In order to make this consideration, it is helpful to consider the relationship between rigid-body joints and members. If the performance of a rigid-body joint were compared to the performance of a rigid member, the differentiating quali- 
ties between a rigid-body joint and a rigid member can be used to differentiate LEM flexures from benchmarks. For instance, a rigid member is infinitely more stiff than a rigid-body joint about the joint's designed axes of rotation or deflection. A rigid-body joint is also infinitely stiff to rotation or deflection about any undesired axis of rotation or deflection, thus providing ideal off-axis stiffness [16]. Additionally, while a rigid-body joint can provide a large range of motion about its designed axis of rotation or deflection, a rigid member does not provide any motion. If parallels are made between a LEM flexure and a rigid-body joint, a LEM flexure should provide similar relative performance to the benchmark, as a rigid-body joint does to a rigid member. Therefore, a LEM flexure should provide less stiffness and greater motion about its designed axis of rotation or deflection, relative to the benchmark, while providing improved off-axis stiffness. Such LEM flexure qualities can then be presented in the form of a relative LEM flexure to benchmark performance criteria, or simply as the LEM flexure criteria, given below:

1. The LEM flexure must have the same or lower flexural/torsional stiffness than the corresponding benchmark.

2. The LEM flexure must provide larger maximum flexural/torsional motion before yielding than the corresponding benchmark.

3. The LEM flexure must exhibit the same or improved off-axis stiffness ratio over the corresponding benchmark.

If a proposed LEM flexure design meets the first and second criterion, but fails to meet the third, the proposed LEM design would not qualify as viable LEM flexure (and may even represent degradation in performance over the benchmark). This is because the off-axis stiffness ratio is an indication on how well the proposed design will perform when subjected to multi-axis loading (as will be explained in further detail later on), which will often be placed on the LEM flexure when it is used in a LEM. Similarly, a proposed LEM flexure design could not be considered as a viable design if it were not to meet either the first or second criteria, while meeting the third.

A brief explanation of supporting knowledge behind each of the three criterions within the LEM flexure criteria is provided next. 

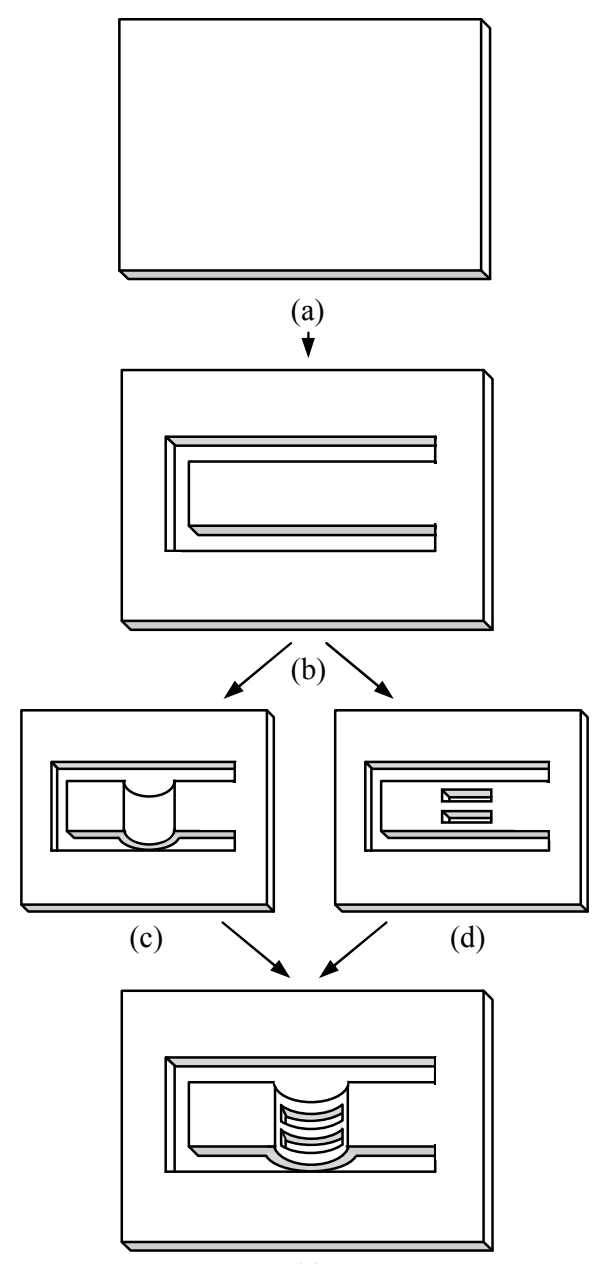

(e)

Figure 2.2: Example of a progression (depicted by arrows) from a (a) lamina to a (b) benchmark and the creation of a (c) LEM flexure created in the benchmark through forming, (d) LEM flexure in the benchmark through removal of material, or otherwise manipulation of the lamina without the use of forming processes, (e) flexure created in the benchmark through both removal of material and forming.

\subsection{Stiffness}

The stiffness of a member is dependent on both geometry and material properties. In bending, rigidity is defined by $E I$, where $E$ is young's modulus, and $I$ is the cross-sectional moment of inertia. For rectangular cross-sections,

$$
I=\frac{b h^{3}}{12}
$$




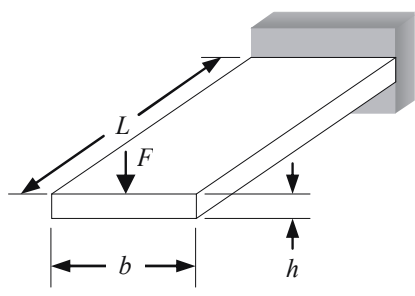

(a)

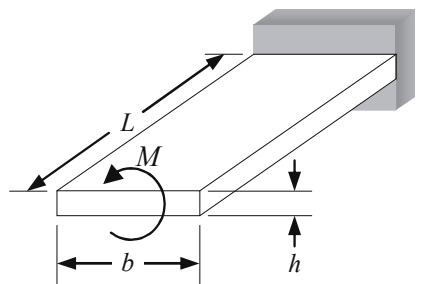

(b)

Figure 2.3: Cantilever beam with rectangular cross-section (the benchmark), loaded in (a) bending and (b) torsion.

In torsion, rigidity is also defined by material properties (the shear modulus, $G$ ) and geometry (polar area moment of inertia, $J$, for closed, circular, cross sections). Because most sheet metal LEMs will have rectangular cross sections, $J$ will be substituted for the parameter $K$, where [17],

$$
K=\frac{1}{16} b h^{3}\left[\frac{16}{3}-\frac{84 h}{25 b}\left(1-\frac{h^{4}}{12 b^{4}}\right)\right]
$$

The flexibility of the cantilever beam in Figure 2.3(a) is expressed as

$$
\delta=\frac{F L^{3}}{3 E I}
$$

and in figure 2.3(b) as,

$$
\theta=\frac{M L}{G K}
$$

\subsection{Yield Strength}

Unlike traditional rigid-body joints, the yield strength of the material sets the upper-boundary of motion for a LEM flexure.

Although the material yield strength is a limiting factor in a LEM flexure's motion, it is apparent that the LEM flexure (through providing lower stiffness, and therefore less resistance to deflection) should provide a larger range of motion than its associated benchmark. 


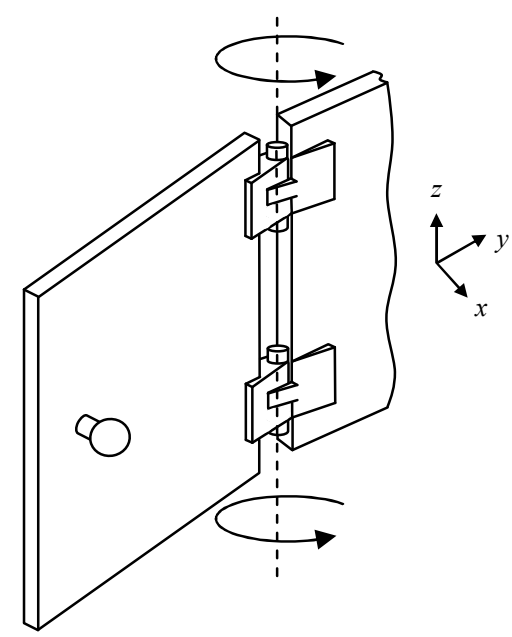

Figure 2.4: A door hinge is an example where off-axis stiffness in rotation about the $x$ or $y$ axes is theoretically infinite.

\subsection{Off-axis Stiffness Ratio}

An important factor for LEM flexure design is how well the LEM flexure behaves in multiaxis loading [9]. A flexure's behavior under multi-axis loading can be compared and contrasted with the behavior of the corresponding benchmark, under the same loading conditions, through the use of off-axis stiffness ratios. An off-axis stiffness ratio is the ratio of the stiffness along an undesired axis, $k_{u}$, over the value of stiffness along the desired axis, $k_{d}$ [16]. Consider the door hinge illustrated in Figure 2.4, with the $z$-axis being the desired axis of rotation. Since the stiffnesses about both the $x$ and $y$ axes ( $k_{x}$ and $k_{y}$ respectively) are essentially infinite, the off-axis stiffness ratios about both the $x$ and $y$ axes, with respect to the $z$-axis, would be: $k_{x} / k_{z}=k_{y} / k_{z}=\infty$.

For a LEM flexure to meet the LEM flexure criteria, the off-axis stiffness ratio for the LEM flexure, $\left(k_{u} / k_{d}\right)_{\text {LEM flexure, }}$, should be a larger value then the off-axis stiffness ratio of the corresponding benchmark, $\left(k_{u} / k_{d}\right)_{\text {benchmark }}$, or $\left(k_{u} / k_{d}\right)_{\text {LEM flexure }}>\left(k_{u} / k_{d}\right)_{\text {benchmark }}$. It is also important to note that though it is ideal for the $\left(k_{u} / k_{d}\right)_{\text {LEM flexure }}>\left(k_{u} / k_{d}\right)_{\text {benchmark }}$ condition to be met throughout the desired range of deflection/rotation, it is most valuable for this condition to be true at the very beginning of motion. This is because the behavior of the LEM flexure under multi-axis loading is most critical at the very start of motion in order to guarantee that the deflection/rotation does not begin about an undesired axis. 


\section{CHAPTER 3. METAL LEM FLEXURES}

\subsection{Forming LEM Flexures from Metals}

Previous research in LEMs has been somewhat material independent [7] and previous LEM flexures have been designed to be made through the removal of material without consideration to the formability of the lamina material [18]. However, forming processes are common operations used in manufacturing parts from sheet metals. Metal LEM flexures can be designed with more complex geometries than have been explored in previous LEM research, particularly through the combination of sheet metal cutting and forming processes (see Figures 2.2(c)-2.2(e)).

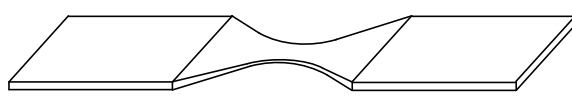

(a)

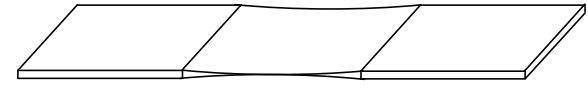

(b)

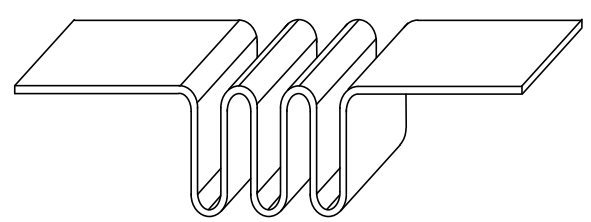

(c)

Figure 3.1: Examples of how sheet metal forming processes can be used to (a) decrease width, (b) decrease thickness and (c) increase length to form a LEM flexure.

Through the use of the sheet metal forming processes, the width and the thickness of the material can be decreased (Figures 3.1(a) and 3.1(b)), while the length can be increased (Figures 3.1(c) and 2.2(b)), all changes of which would decrease the stiffness of the potential LEM flexure (see equations 2.1-2.4). The cross-sectional profile can also be formed to provide greater off-axis stiffness (Figure 3.2). 


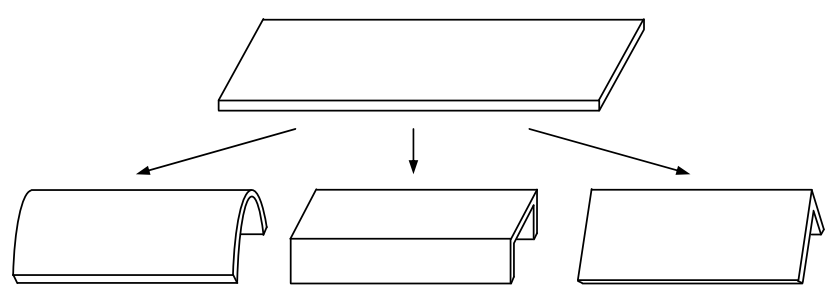

Figure 3.2: Examples of how sheet metal forming process can be used to increase the cross-section moment of inertia through changing the cross-sectional profile of the benchmark.

\subsection{Other Advantages of Metal LEMs}

Besides their formability, there are also several other advantages that metals in LEMs have over other materials depending on the application.

In manufacturing, there are relatively inexpensive stamping processes current used to fabricate sheet metal parts that could be used to mass produce metal LEMs at a low cost. A complete metal LEM could be cut and formed from a single sheet of metal with single stamp. This concept could be extended to included arrays of LEMs that could be made with a single stamp.

Along with the manufacturing advantage of metal LEMs, metal LEMs also have important mechanical characteristics that are inherent to all compliant mechanisms made from metals. A list of these mechanical characteristics is given by Howell and is provided below for convenience [8]. That is, metals should also be considered in LEMs for the following reasons:

- Predictable material properties

- Good performance in high-temperature environments

- Biocompatibility of some metals

- Low susceptibility to creep

- More predictable fatigue life

- Ability to operate in many harsh environments

- Electrical (and thermal) conductivity 


\section{CHAPTER 4. METAL LEM FLEXURE FOR BENDING: REVOLUTE U-FORM FLEX-}

URE (RUFF)

Now that a LEM flexure criteria has been established, and the advantages of metal LEMs have been discussed, these two concepts can be combined to produce viable metal LEM flexure designs.

Dai and Cannella refer in their research to a cardboard carton crease as a "die-pressed groove line on a cardboard to enable a carton to be erected and folded into a certain structure" [19]. A kinematic model of the folding of a cardboard carton is then established with carton creases analyzed as equivalent revolute joints [19]. The concept of a die-pressed groove into cardboard could be readily transferred to sheet metals where die-pressing is commonly used in various forming processes. These die-pressed grooves could then be kinematically analyzed as revolute joints with varying stiffness characteristics depending on the material and geometry of the die-pressed grooves. This concept of die-pressed groves is the foundation for the Revolute U-Form Flexure (RUFF) (shown in Figure 4.1(a)). The RUFF is designed to act as a metal LEM flexure.

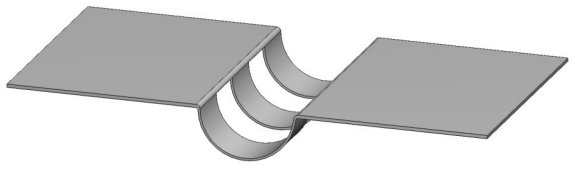

(a)

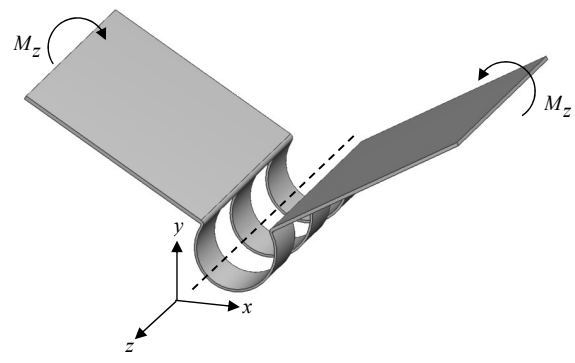

(b)

Figure 4.1: Revolute U-Form Flexure (RUFF) in (a) deflected and (d) undeflected positions.

The geometry of the RUFF has been specifically designed to provide a localized decrease in stiffness towards an end bending moment (see Figure 4.1(b)). When forming the die-pressed, 


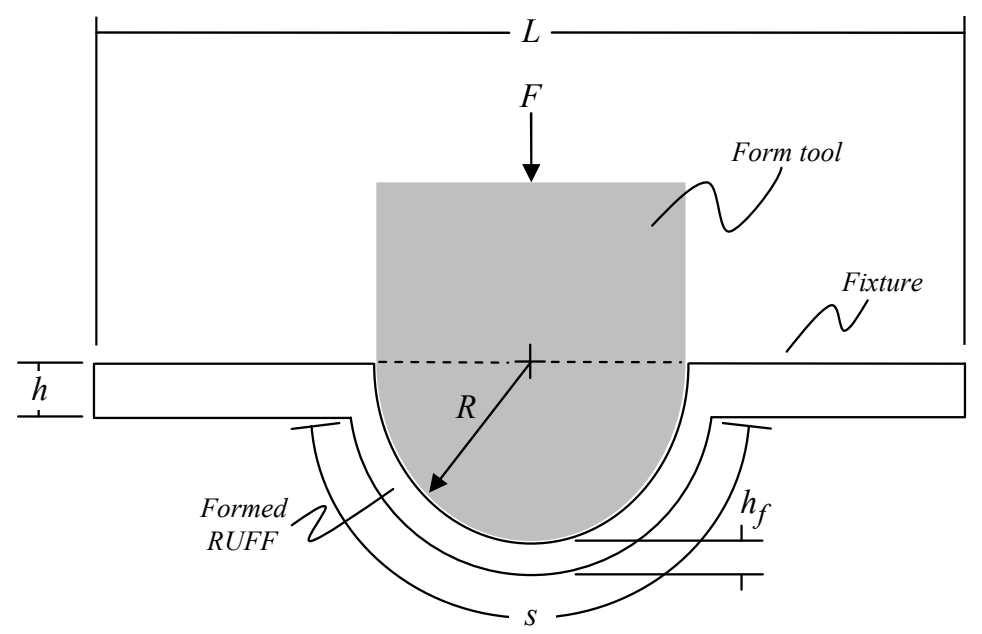

Figure 4.2: Schematic of post-formed RUFF.

or u-shaped, groove into the benchmark, the benchmark length, $L$, is designed to remain constant to locally constrain the material length to increase, and the thickness, $h$, to decrease (see Figure 4.2). This localized increase in length and decrease in thickness causes a decrease in stiffness (see equation 2.3) and decrease in $I_{z}$ (see Figure 4.1(b) and equation 2.1), respectively. Also, the longitudinal slots that are cut in the die-pressed grove provide a reduction in the width, $b$ (see Figure 2.3(a)). This reduction in $b$ provides a directly proportional reduction in $I_{z}$ (see Figure 4.1(b) and equation 2.1). Therefore, the combined effect of the localized decrease in thickness and width, and increase in length provides a decrease in stiffness and increase in flexibility. To design against undesirable off-axis stiffness ratios, consideration was made to not limit the localized width on the outer edges of the RUFF, but that the width be reduced in the internal localized thickness only.

\subsection{RUFF Analysis}

To analyze the RUFF relative to the LEM flexure criteria, the post-formed length, $s$, and thickness, $h_{f}$, must first be calculated in terms of the known parameters: $h$, and form tool radius, $R$ (see Figure 4.2). Therefore, $s$ is approximated as,

$$
s=\pi R
$$




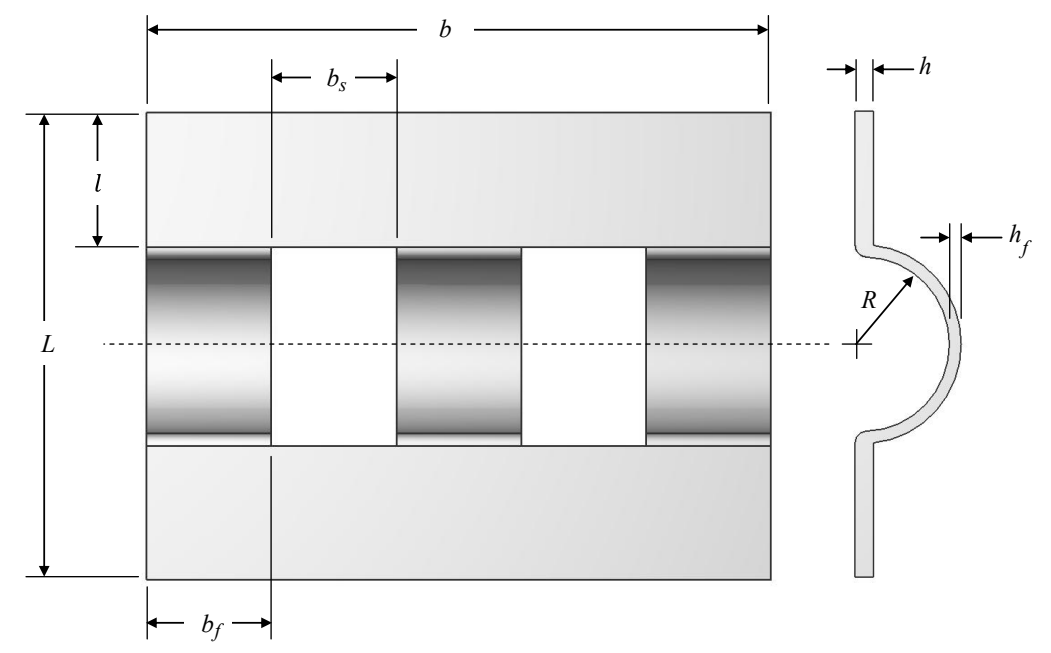

Figure 4.3: Schematic of RUFF fabrication set-up.

and if $b$ is assumed to remain constant while the RUFF is formed, the condition $s h_{f}=2 R h$ would be true, allowing $h_{f}$ to be solved for (see equation 4.2).

$$
h_{f}=2 R \frac{h}{s}
$$

Equation 4.2 enables the analysis of the stiffness characteristics of the RUFF. In this evaluation, three methods are used in order to provide confidence in the validity of the analysis results. These three methods are: closed-form solution, finite element analysis (FEA), and physical prototype testing. The material and geometry used to generate both the closed-form and FEA models are identical to that of the prototype.

Copper sheet metal was chosen for the lamina material (with $E=16 \times 10^{6} \mathrm{psi}$ ) because the ideal manufacturability of copper for the purpose of prototyping. 24 gauge was chosen because it is readily available from suppliers and provides a thickness that is easily formed, and requires relatively low forces to deflect. The forming tool radius, $R$, was selected so that it would provide a reasonable amount of increase in length and decrease in thickness in the benchmark to provide a relatively noticeable decrease in stiffness over the benchmark. Therefore, the dimension for $R$ was driven by the original thickness of 24 gauge sheet metal copper. All the dimensions used in the three analysis methods for the RUFF are called-out in Figure 4.3, and the values for each of these dimensions are given in Table 4.1. 
Table 4.1: RUFF prototype dimensions

\begin{tabular}{cc} 
Name & Value(in) \\
\hline$L$ & 1.00 \\
$l$ & 0.20 \\
$b$ & 2.00 \\
$b_{f}$ & 0.40 \\
$b_{s}$ & 0.40 \\
$h$ & 0.020 \\
$h_{f}$ & 0.013 \\
$R$ & 0.30
\end{tabular}

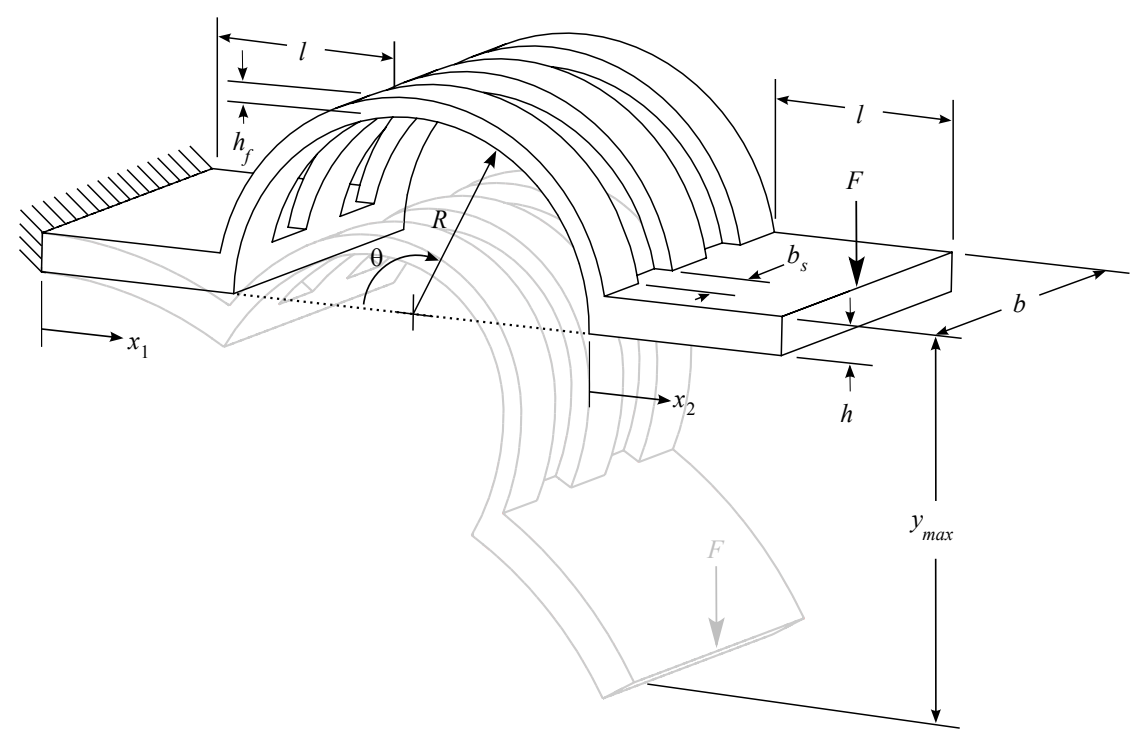

Figure 4.4: Both the undeflected and deflected positions of the full RUFF closed-form model.

\subsubsection{RUFF Closed-form Solution}

To derive a closed-form force-deflection/stiffness solution for the RUFF, boundary conditions were used that could provide a basic understanding of the stiffness characteristics of the RUFF and could be easily duplicated in the testing of a RUFF prototype. As shown in Figure 4.4, the RUFF was analyzed with one end of the beam fixed and a non-follower, axially-perpendicular force place on the opposite end. 
Since the RUFF is designed specifically for metals, a linear solution was assumed to be sufficient to model force-deflection characteristics of the RUFF before yield and Castigliano's theorem was used to model the RUFF deflection [20]. With the boundary conditions shown in Figure 4.4 Castigliano's theorem results in:

$$
y_{i}=\frac{\partial U}{\partial F_{i}}=\int \frac{1}{2 E I}\left(M \frac{\partial M}{\partial F_{i}}\right) d x
$$

where $y_{i}$ is the deflection caused by the input force $F_{i}$ (with the subscript $i$ indicating that both the deflection and the force are directed along the same axis), $U$ is the total strain energy, and $M$ is the internal moment within the RUFF.

To apply equation 4.3 to the RUFF, it must be broken up in to three parts (two straight cantilever beams, and one initially curved cantilever beam) and the deflections for each calculated separately. The deflection, $y_{\max }$, for the whole RUFF model (shown in figure 4.4) can then be solved for by combining the solutions for three parts of the RUFF together. For this approach to provide a solution, the internal moment equations for each of the beams must be determined as functions of a variable location along the length of the beam. With reference to the variables of integration, $x_{1}, \theta$, and $x_{2}$ (shown in Figure 4.4), the internal moment equations for each of the three sections of the beam are:

$$
\begin{gathered}
M\left(x_{1}\right)=F\left(L+2 R+x_{1}\right) \quad(0 \leq \theta \leq l) \\
M(\theta)=F[L+R(1-\cos \theta)] \quad(0 \leq \theta \leq \pi) \\
M\left(x_{2}\right)=F x_{2} \quad\left(0 \leq x_{1} \leq l\right)
\end{gathered}
$$

Before the integrations are carried out, the equation for $y_{\max }$ would be

$$
y_{\max }=\frac{1}{2 E I_{s}}\left(\int_{0}^{l} M\left(x_{1}\right) \frac{\partial M\left(x_{1}\right)}{\partial F} d x_{1}+\int_{0}^{l} M\left(x_{2}\right) \frac{\partial M\left(x_{2}\right)}{\partial F} d x_{2}\right)+\frac{1}{2 E I_{c}} \int_{0}^{\pi} M(\theta) \frac{\partial M(\theta)}{\partial F} d \theta
$$




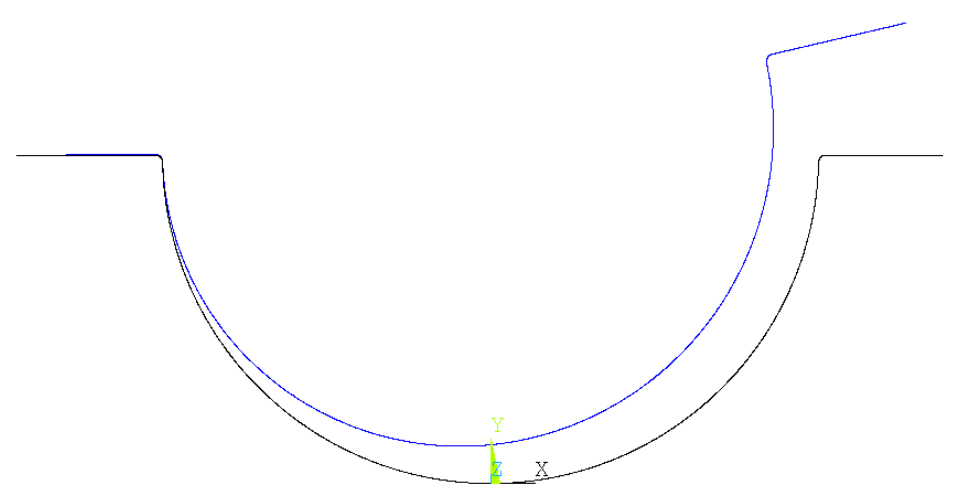

Figure 4.5: An FEA of rendering of the RUFF in both deflected and undeflected positions.

By combining equations 4.4-4.6 with equation 4.7, and carrying out all integrations, $y_{\max }$ can be put in terms of known parameters, as shown in equation 4.8 below,

$$
y_{\text {max }}=\frac{F}{E}\left[\frac{1}{2 I_{c}}\left(\pi l^{2}+2 \pi l R+\frac{3}{2} \pi R^{2}\right)+\frac{L}{I_{S}}\left(\frac{4}{3} l^{2}+3 l R+2 R^{2}\right)\right]
$$

where $I_{C}$ and $I_{S}$ are the moments of inertia for the initially curved and straight sections. In reference to parameters in Figure 4.4, $I_{S}$ is equal to equation 2.1. Due to the reduced width (dependent on the slot width $b_{s}$ ) and decrease thickness, $h_{f}$, in the initially curved section, $I_{c}$ for the initially curved section is

$$
I_{c}=\frac{\left(b-n b_{s}\right) h_{f}^{3}}{12}
$$

where $n$ is the number of slots cut in the benchmark (i.e. in figures 4.4 and $4.3 n=2$, which, is the same $n$ used in the RUFF prototype and therefore in the closed-form and FEA models).

\subsubsection{RUFF FEA Model}

The RUFF FEA model uses the same boundary and loading conditions specified in the RUFF closed-form solution (see Figure 4.4). For the FEA model, the RUFF was modeled with twodimensional non-linear beam elements (ANSYS ${ }^{\circledR}$ beam 3 elements) with three degrees of freedom at each node: translation along the $x$ and $y$ axes and rotation about the $z$-axis (see Figure 4.1(b) for a reference on axes). An FEA rendering of the RUFF in both the deformed and undeformed positions is given in Figure 4.5. 


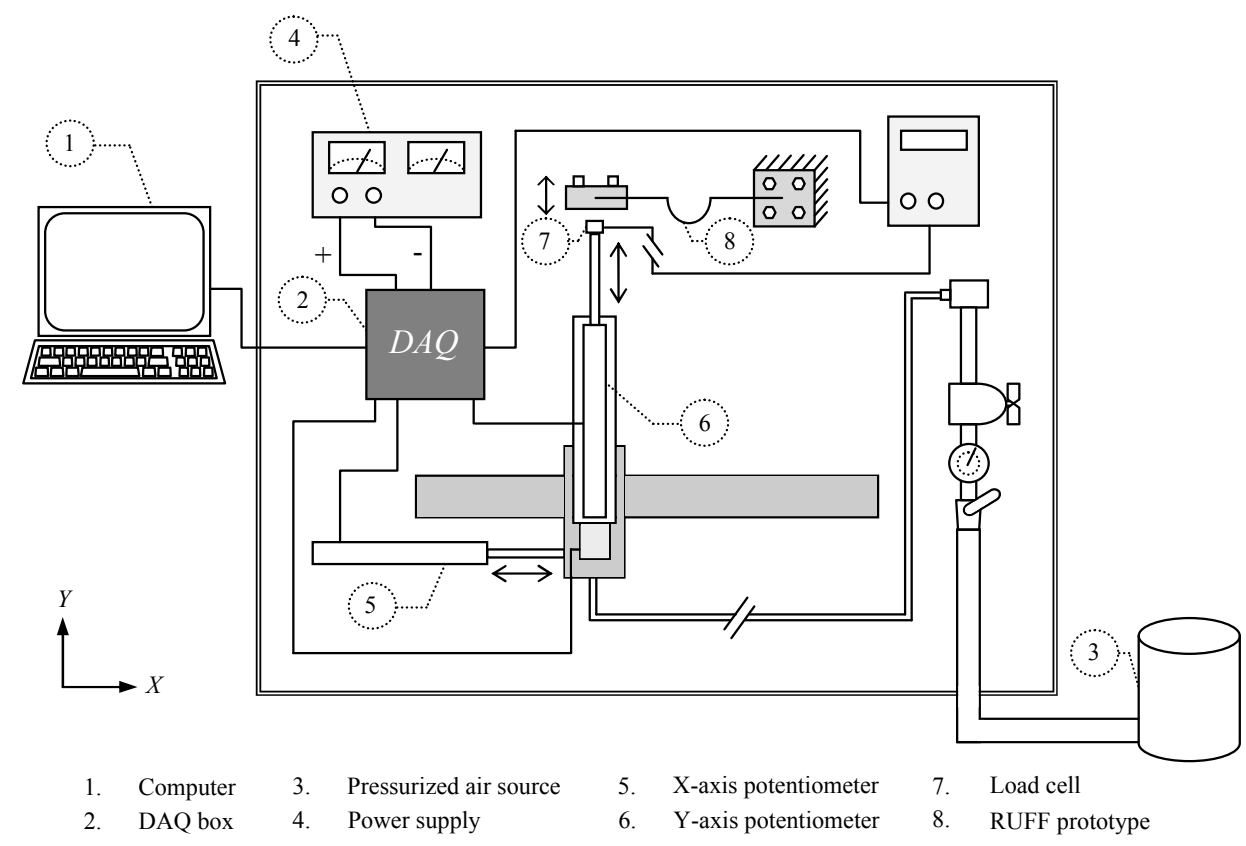

Figure 4.6: Schematic of force-deflection tester used to determine the stiffness characteristics of the RUFF prototype.

\subsubsection{RUFF Prototype Manufacture and Testing}

The prototype for the RUFF was fabricated using manufacturing processes (such as milling and draw-forming) that are not desirable for mass LEM production, yet were suitable for a singlebuild prototype. The RUFF prototype is shown in Figure 4.7(a). Ideally, in a mass production scenario, the slots in the RUFF would be manufactured through a RUFF punching/blanking process, and stretch formed to the desired radius.

Once manufactured, the RUFF prototype was placed in the custom force-deflection tester (see figure 4.6) under approximately the same loading conditions that were used in both the closedformed and FEA models. The force-deflection tester used a stepper motor to extend a shaft to create the desired input deflection on the RUFF prototype. A load cell, attached at the end of the extending shaft, was used to collect force data, while other instrumentation was used to record the distance that the shaft had extended the deflection of the RUFF. Figure 4.7(b) shows a RUFF prototype deflected in the force-deflection tester. 


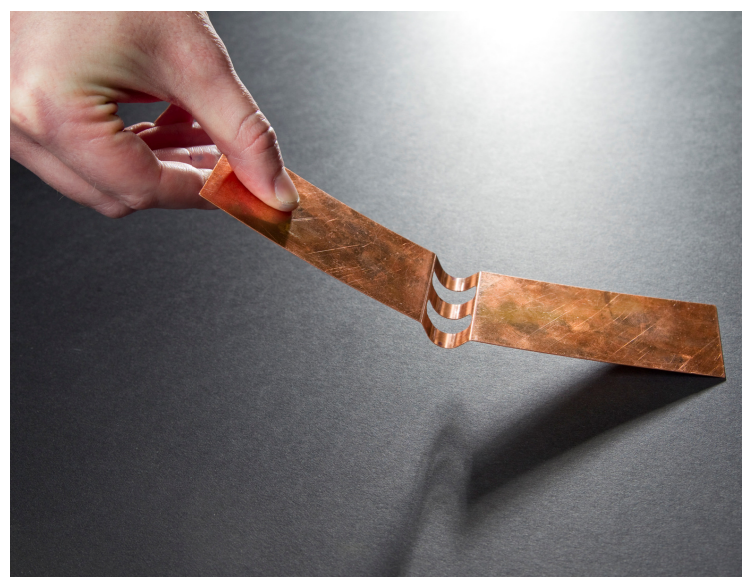

(a)

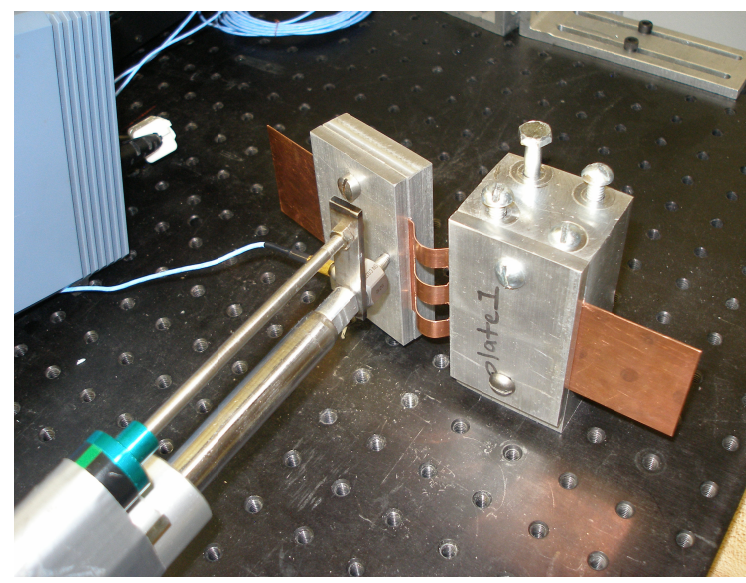

(b)

Figure 4.7: Photographs of (a) the RUFF prototype and (d) the RUFF prototype deflected in the force-deflection tester.

\subsection{RUFF Versus Benchmark: Force-deflection}

Evaluation of the RUFF closed-form solution, FEA simulation, and prototype test data are plotted in

Figure 4.8 provides a force-deflection plot of the RUFF closed-form solution, FEA simulation, and prototype test data along with a force-deflection plot of the benchmark. The forcedeflection data is cut-off at the point where the maximum equivalent stress, $\sigma_{\max }$, has exceeded the material yield stress, $\sigma_{\text {yield }}(40 \mathrm{ksi}$ for the copper prototype).

The closed-form, FEA, and prototype curves show similar linear behavior before the material yield point is reached, a non-linear trend in the FEA and prototype data at points close to yield. For the particular geometry and material chosen for this analysis, the closed-form solution and FEA model can be reasonable predictive models for the stiffness behavior of the RUFF with stresses under $\sigma_{\text {yield }}$.

In regards to the LEM flexure criteria, the particular geometry and material for this analysis, Figure 4.8 displays that the RUFF has improved performance over the benchmark in accordance to the first and second LEM criteria. The slope for the benchmark is approximately eight and a half times greater than the RUFF, representing higher localized flexibility provided by the RUFF. The RUFF also provides a deflection before yield that is approximately three times greater than the benchmark, satisfying the second LEM flexure criterion. 


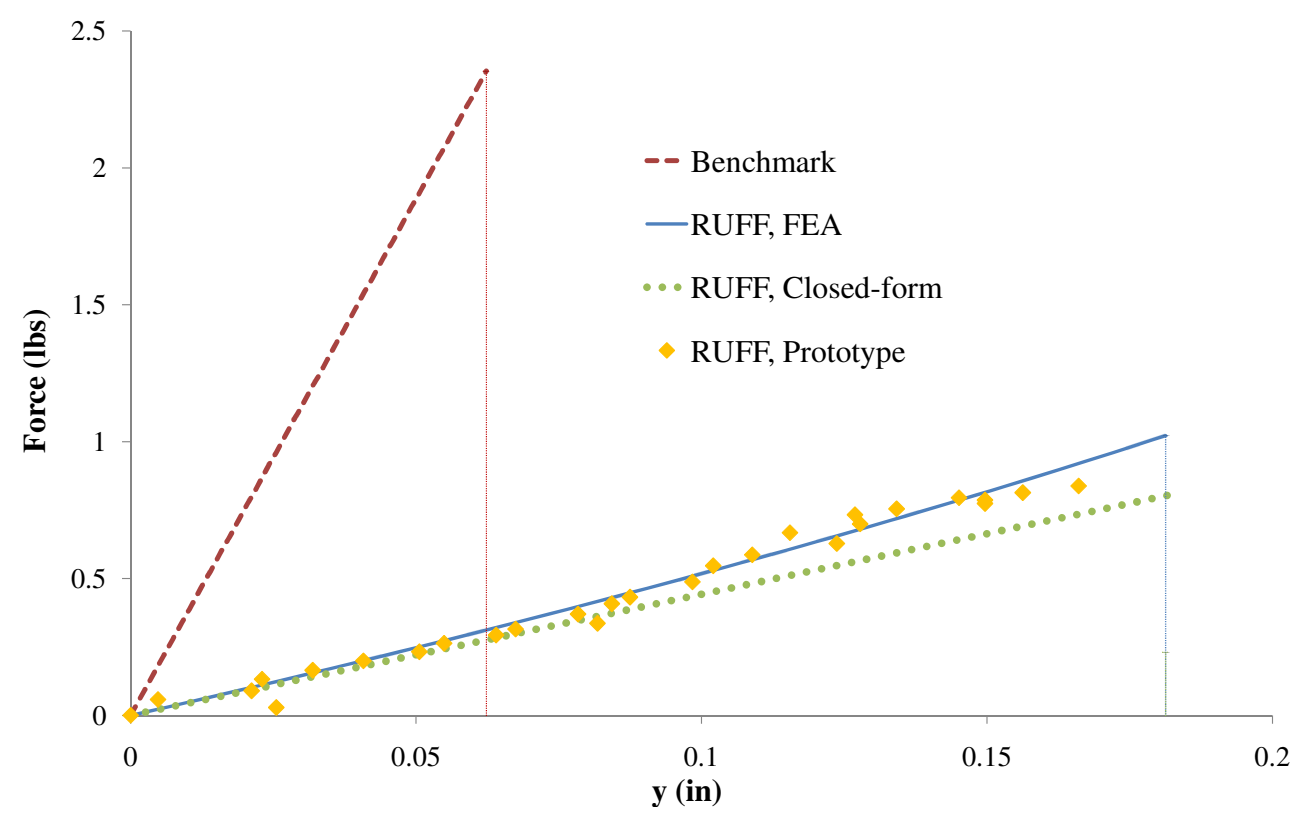

Figure 4.8: Force-deflection plot of the RUFF closed-form solution, FEA simulation, and prototype test data along with a force-deflection plot of the benchmark. The vertical dotted-lines emphasize the point at which the maximum equivalent stress $\left(\sigma_{\max }\right)$ has exceeded the material yield stress $\left(\sigma_{y i e l d}\right)$

Figure 4.8 only provides force-deflection characteristics of a particular configuration. The closed-form deflection solution for both the RUFF and benchmark can be non-dimensionalized to provide a more generalized force-deflection solution set. This can be done by dividing $y_{\max }$ by the overall length $L$ to generate non-dimensionalized deflection values. To non-dimensionalized the input force, the non-dimensionalized force parameter, $\alpha^{2}$, is used:

$$
\alpha^{2}=\frac{F L^{2}}{E I_{s}}
$$

Non-dimensionalization of the force-deflection data shown in Figure 4.8 is shown in figure 4.9. As in Figure 4.8, Figure 4.9 displays a stiffness for the benchmark that is approximately eight and half times greater than the RUFF stiffness.

\subsection{RUFF Versus Benchmark: Off-axis Stiffness Ratio}

Through the stiffness characteristics analysis, it has been established that the RUFF design can successfully meet the first and second LEM flexure criterion. However, an off-axis stiffness 


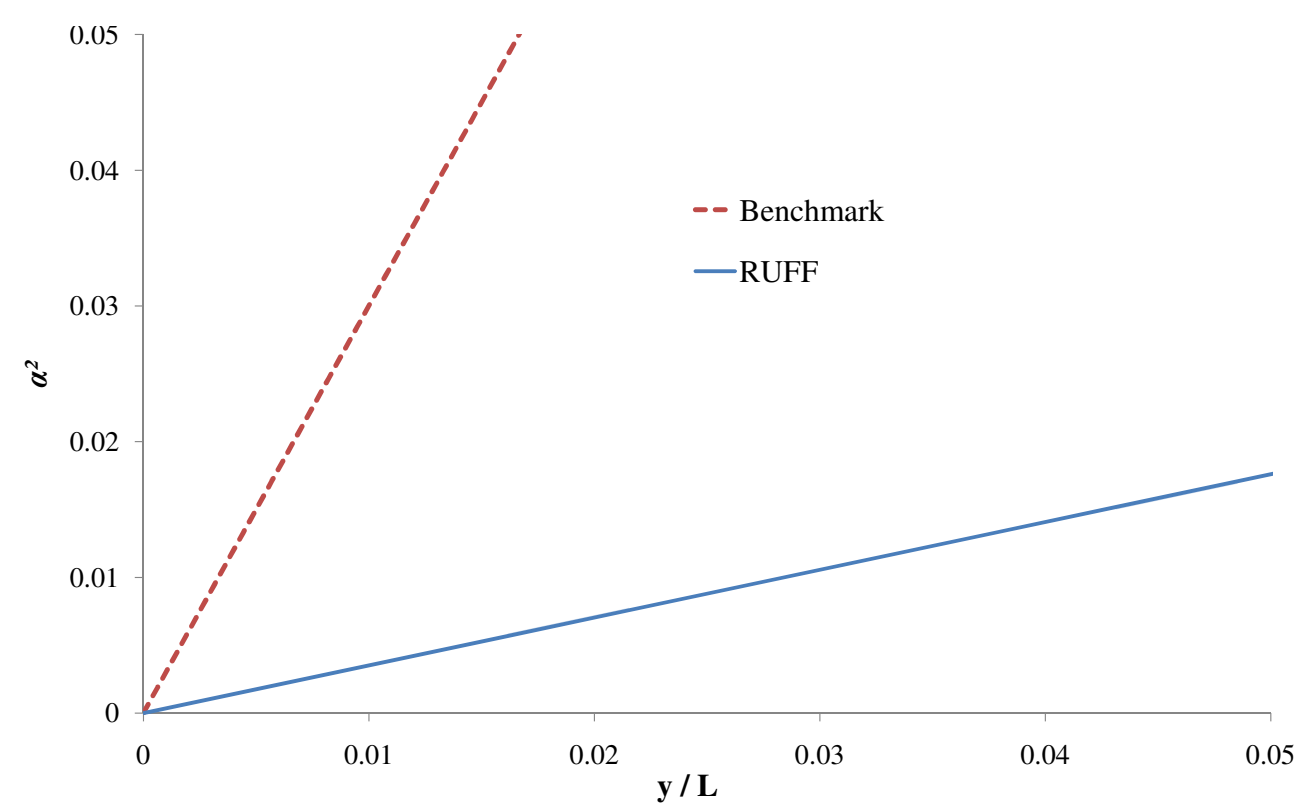

Figure 4.9: Force-deflection plot of non-dimensionalized RUFF data.

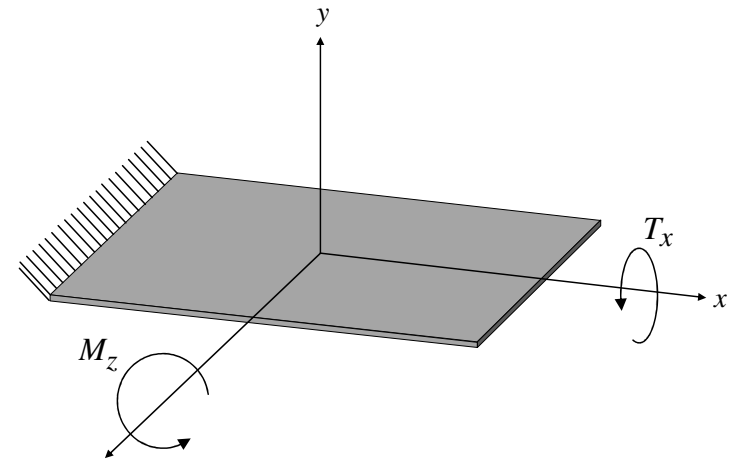

(a)

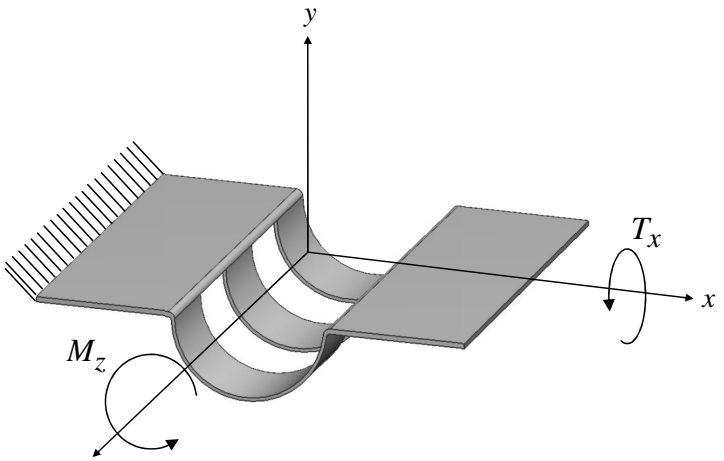

(b)

Figure 4.10: Representation of desired (moment, $M_{z}$ ) and undesired (torsion, $T_{x}$ ) loading shown on both the (a) benchmark and the (b) RUFF.

ratio analysis is required to determine how well the RUFF performs in multi-axis loading, and if the RUFF meets the off-axis stiffness ratio requirement established by the third LEM flexure criterion.

Figure 4.10(b) depicts the ideal motion for the RUFF, which is a rotation about the $z$-axis. The undesired axis of rotation, are the $x$ and $y$ axes. Due to the lengthening of the RUFF along the $x$-axis, the $x$-axis has been determined to be the undesired axis of most interest. To determine the comparative stiffness of the RUFF in multi-axis loading, the ratios of the stiffness when subjected 


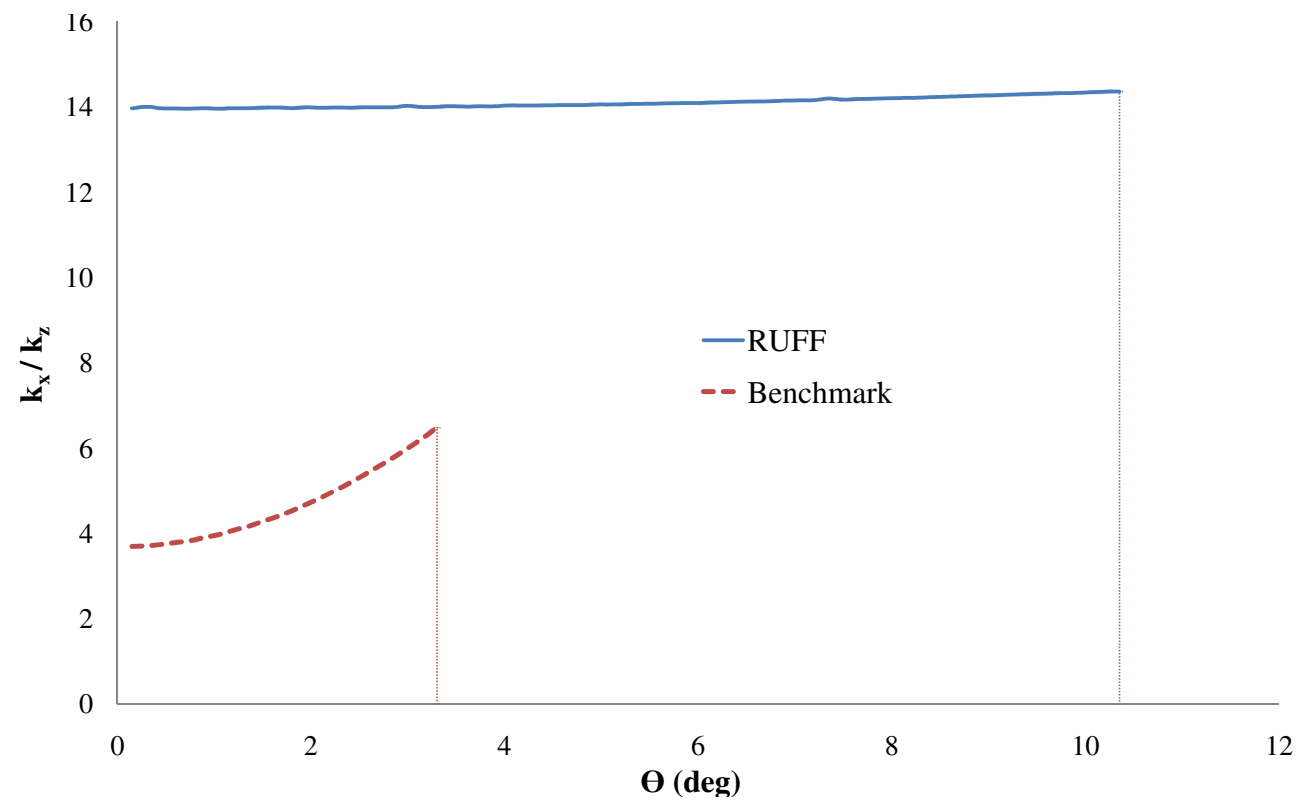

Figure 4.11: Comparison of off-axis stiffness ratio between the RUFF and benchmark.

to a torque $T_{x}\left(k_{x}\right)$ to stiffness when subjected to a moment $M_{z}\left(k_{z}\right)$, is compared to the benchmark, as shown in figure 4.11.

An FEA model was used to calculate the off-axis stiffness ratio $k_{x} / k_{z}$. The FEA model was subjected to angular displacements, $\theta$, about both the $z$ and $x$ axes and the reaction loads $M_{z}$ and $T_{x}$ were calculated. Once the reaction loads $M_{z}$ and $T_{x}$ were known, $k_{x}$ and $k_{z}$ were determined by dividing $T_{x}$ and $M_{z}$ by $\theta$, or, $T_{x} / \theta=k_{x}$ and $M_{z} / \theta=k_{z}$. Figure 4.11 provides a plot of the $k_{x} / k_{z}$ at a varying $\theta$ for the RUFF and the benchmark. The plotted data is shown up to the point where the material yield stress has been exceeded.

The comparison of off-axis stiffness ratios for the RUFF and benchmark show that, at a $\theta$ of approximately zero, the RUFF has an off-axis stiffness ratio approximately three and a half times greater than the benchmark. This difference in off-axis stiffness ratios slightly decreases as the benchmark approaches its maximum deflection, however, the RUFF displays a higher offaxis stiffness ratio throughout the range of motion and the RUFF improves performance in multiaxis loading over the base performance of the benchmark. Therefore this RUFF configuration successfully meets the third LEM flexure criterion. 


\subsection{RUFF Conclusion}

The RUFF successfully meets and exceeds the LEM flexure criteria and, therefore, qualifies as a viable metal LEM flexure. Thus, the RUFF provides a designer with an addition tool in the design of metal LEMs and, by so doing, assists in the application of LEMs to design functions, environments, and cost-effective manufacturing processes to which LEMs could previously not easily be considered. 


\section{CHAPTER 5. METAL LEM FLEXURE FOR TORSION: TORSIONAL U-FORM FLEXURE (TUFF)}

To design a LEM flexure that would perform well in torsion, consideration was made into reducing the width, $b$, to decrease the polar moment of inertia $K$, and in turn increase the torsional rotation, $\theta$ (see figure 2.3(b) and equations 2.2 and 2.4). To reduce $b$, transverse slots were designed in the middle of the flexure. Decrease in $b$ to reduce torsional stiffness also increases stiffness in bending, causing poor performance in multi-axis loading. To compensate for this, a radius was designed in the cross-sectional profile to increase the moment of inertia in bending (see Figure 3.2). The combination of width reduction and cross-sectional profile forming results in the Torsional U-form Flexure (TUFF) shown in Figure 5.1(a). For the TUFF to be placed in line with flat LEM members, the flexure design also includes transitional areas (where the cross-sectional profile transitions from curved to flat) on both ends.

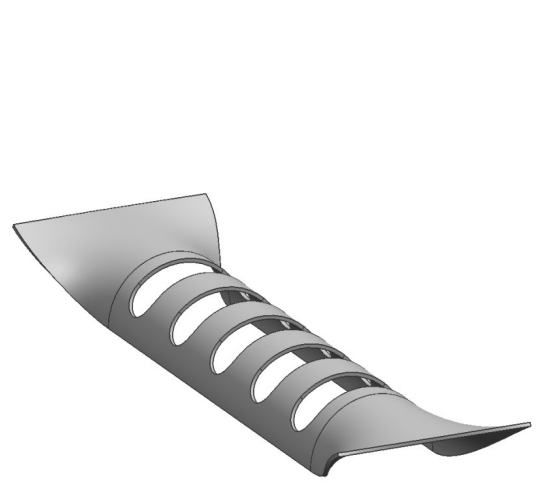

(a)

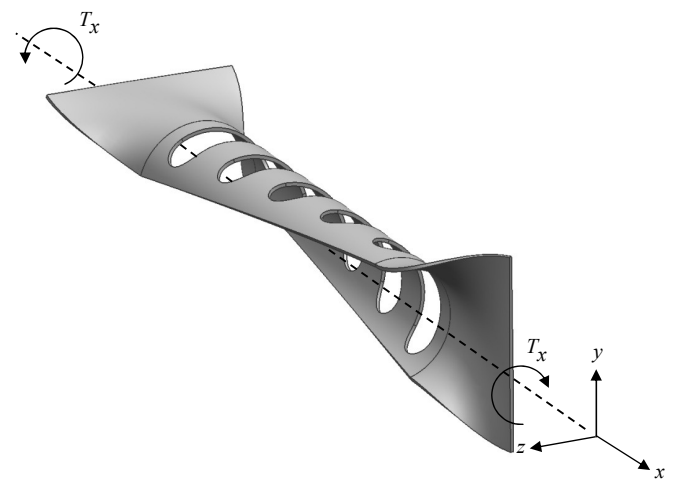

(b)

Figure 5.1: Torsional U-Form Torsional (TUFF) in (a) deflected and (d) undeflected positions. 


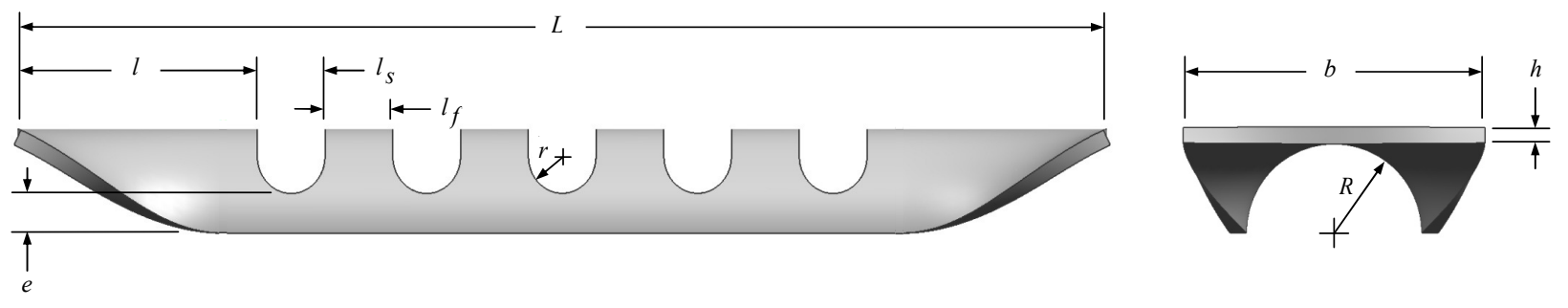

Figure 5.2: FEA deformation plot of TUFF rotated 45 degrees under end torque.

Table 5.1: TUFF prototype dimensions

\begin{tabular}{cc} 
Name & Value(in) \\
\hline$L$ & 6.50 \\
$l$ & 2.13 \\
$l_{s}$ & 0.25 \\
$l_{f}$ & 0.25 \\
$b$ & 1.50 \\
$h$ & 0.020 \\
$e$ & 0.18 \\
$R$ & 0.46 \\
$r$ & 0.13
\end{tabular}

\subsection{TUFF Analysis}

Both FEA and physical prototype testing were used to provide data needed to compare the TUFF against the LEM flexure criteria. A closed-formed solution for the TUFF was not derived due to the profile transition that occurs in the length of the TUFF (dimensioned as $l$ in figure 5.2).

The material and geometry used to generate the FEA model matches that of the prototype. As with the RUFF, the material used was 24 gauge sheet metal copper. The dimensions where chosen so that the TUFF prototype could be fairly easy to manufacture, and still provide a reasonable improvement over the benchmark. The dimensions for the TUFF are specified in Figure 5.2, and the values for each of these dimensions are given in Table 5.1. 


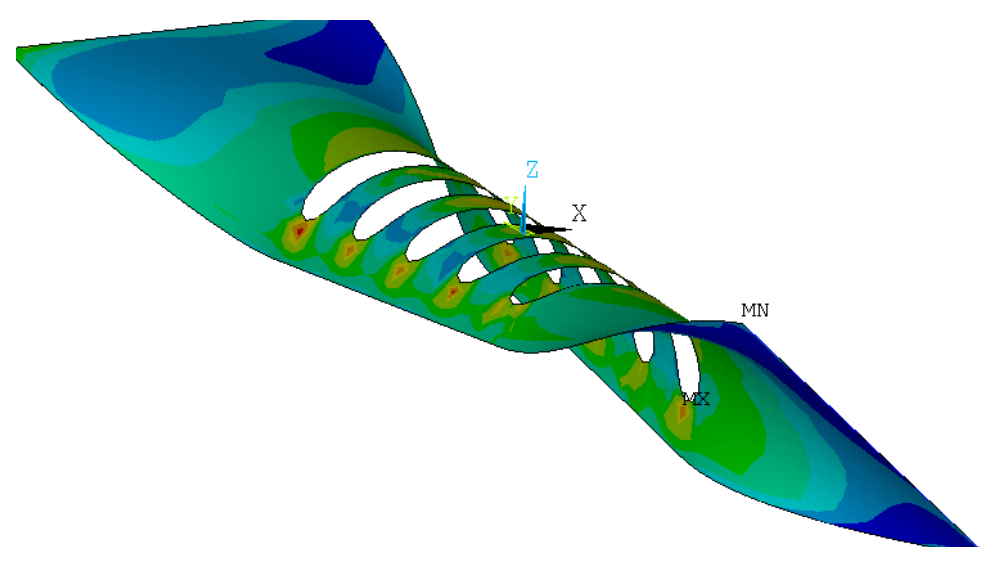

Figure 5.3: FEA equivalent stress plot of TUFF displaced through $45^{\circ}$ of rotation.

\subsubsection{TUFF FEA Model}

To model the TUFF in FEA, boundary conditions were selected that could provide both a basic understanding of the stiffness characteristics of the TUFF, and that could be readily tested with a RUFF prototype. The TUFF was fixed at one end, while a rotational displacement was applied at a node in the center of the opposite end. A rotational displacement was applied at this center node and the node was also fixed so that it could not translate in any direction, but it was free to rotate about the $x$-axis (see Figure 5.2). The nodes at the outer corners of the rotationally displaced end were fixed so that they could not translate in the $x$-direction. This additional boundary condition was included so that the shear forces (due to the reactive torque cause by the rotation displacement) would not be allowed to draw in the sides of the TUFF towards the fixed end, as this would likely not be permissible if the TUFF were used in a LEM.

To create the TUFF FEA model, a three-dimensional TUFF surface was modeled and then meshed with ANSYS ${ }^{\circledR}$ shell 93 elements. These elements were chosen because they have six degrees of freedom at each node and can handle non-linear deflections and strains. A FEA equivalent stress rendering of the TUFF, displaced through $45^{\circ}$ of rotation, is given in figure 5.3.

\subsubsection{TUFF Prototype Manufacture and Testing}

The manufacturing processes used to fabricated the TUFF prototype were suitable for a single-build prototype (see figure 4.7(a) for a photograph of the RUFF prototype) but not necessarily suited for mass production. Copper tubing was lathed to have a wall thickness equal to 24 


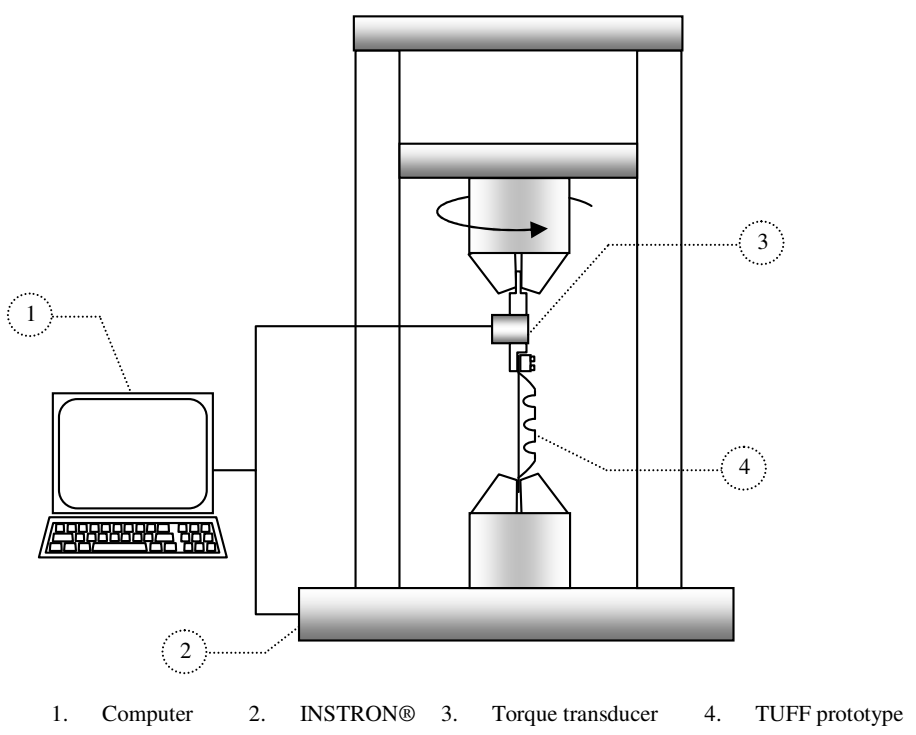

Figure 5.4: Schematic of torque-rotation tester set-up used to determine the stiffness characteristics of the TUFF prototype.

gauge sheet metal copper thickness, transverse slots where cut in the tube and then the tube was cut in half (all with wire-EDM), and each end flattened to create the desired geometry. In mass production, the slots in TUFF would be manufactured through a punching/blanking process, and then draw formed to the required $\mathrm{u}$-shaped profile radius.

Once manufactured, the TUFF prototype was placed in an INSTRON ${ }^{\circledR}$ device set-up to act as a torque-rotation tester (see figure 5.4). The TUFF prototype was placed under approximately the same loading conditions that were modeled in the FEA model. A torque transducer, attached in series to the TUFF prototype, was used to collect the reaction torque while the TUFF was rotationally deflected. Figure 5.5(b) provides a photograph of the TUFF prototype rotationally deflected in the torque-rotation tester.

\subsection{TUFF Versus Benchmark: Torque-rotation}

The FEA model and the prototype test data provide two distinct data sets to describe the torque-rotation characteristics of the TUFF. A plot of these data sets is given in Figure 5.6 and compared to the torque-rotation plot of the benchmark. The plot is cut off at the point where the maximum equivalent stress, $\sigma_{\max }$, has exceeded the material yield stress, $\sigma_{\text {yield }}(40 \mathrm{ksi})$. 


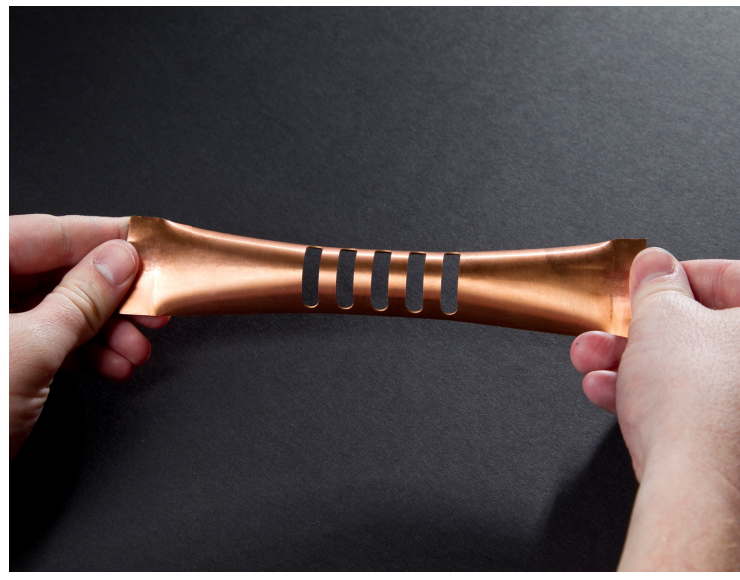

(a)

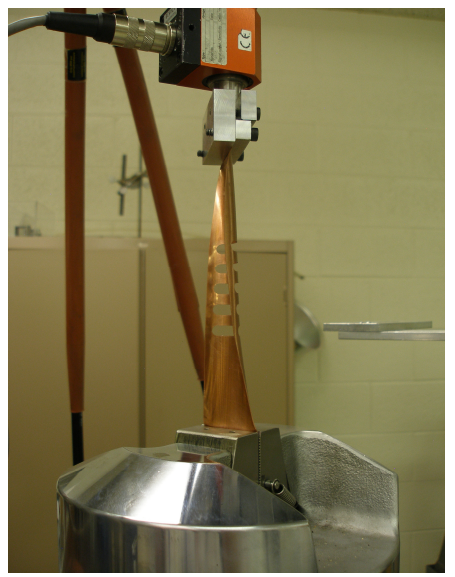

(b)

Figure 5.5: Photographs of (a) the TUFF prototype and (d) the TUFF prototype deflected in the torque-rotation tester.

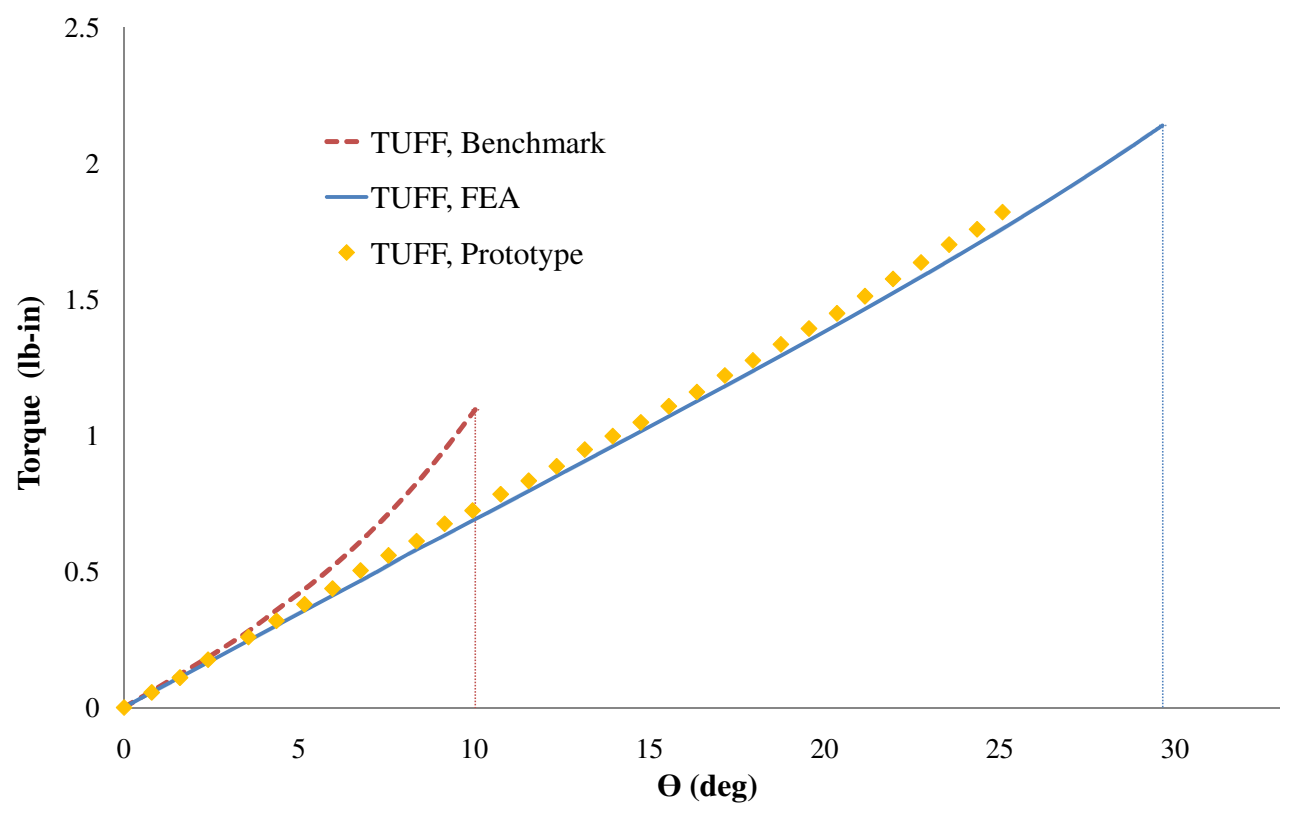

Figure 5.6: Torque-rotation plot of the TUFF FEA simulation and prototype test data along with a torque-rotation plot of the benchmark. The vertical dotted-lines emphasize the point at which the maximum stress $\left(\sigma_{\max }\right)$ in the LEM profile or TUFF has reached the material yield stress $\left(\sigma_{\text {yield }}\right)$.

The TUFF torque-rotation FEA and prototype curves all show similar linear behavior before the material yield point is reached. For the particular geometry and material chosen, the FEA model is an accurate predictive model for the stiffness behavior of the TUFF within the elastic range of motion. 


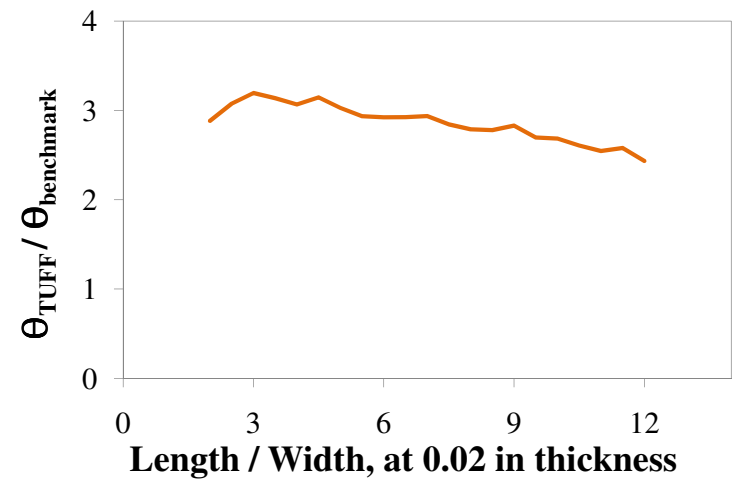

(a)

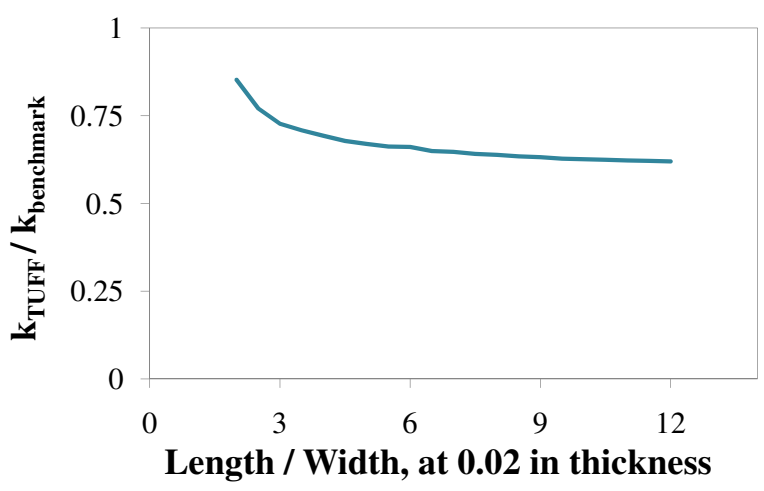

(c)

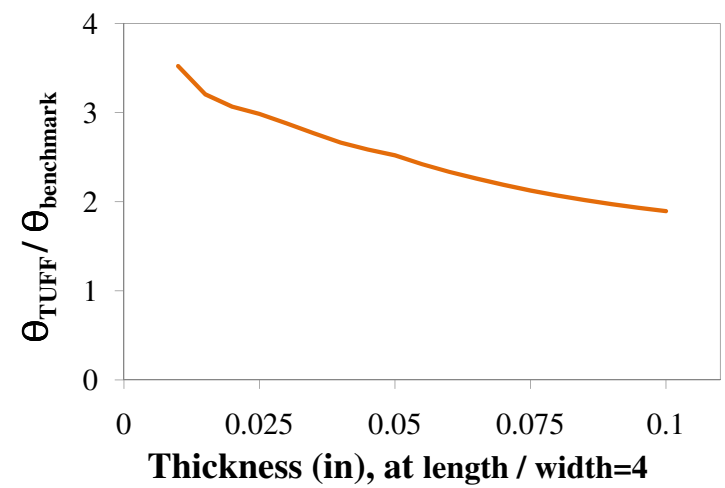

(b)

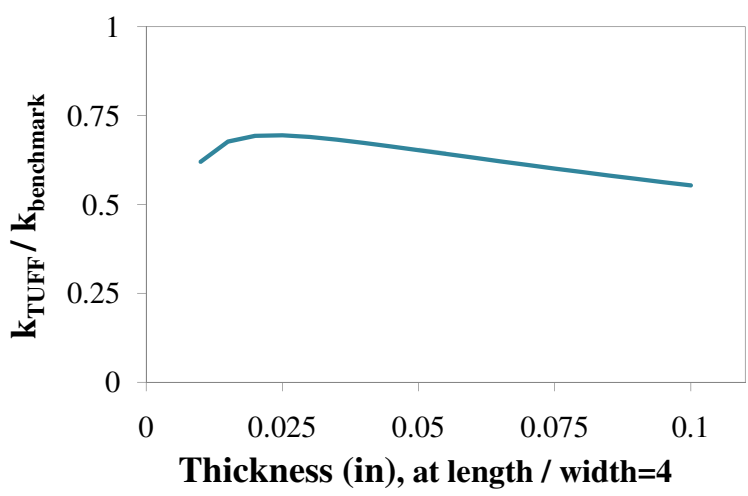

(d)

Figure 5.7: Plots of: The change in the ratio of the maximum rotation of the TUFF, $\theta_{T U F F}$, over the maximum rotation of the benchmark, $\theta_{\text {benchmark }}$, as (a) the ratio of length-over- width is varied with the thickness held constant and as (b) thickness is varied and the length-over-width ratio is held constant. As well as the change in the ratio of the stiffness of the TUFF, $k_{T U F F}$, over the stiffness of the benchmark, $k_{\text {benchmark }}$, as (c) the ratio of length-over- width is varied with the thickness held constant and as (d) thickness is varied and the length-over-width ratio is held constant.

In regards to the LEM flexure criteria, for the particular geometry and material for this analysis, Figure 5.6 displays that the TUFF has improved performed over the benchmark in accordance to the first and second LEM flexure criterion. At low rotations the stiffness for the TUFF and benchmark are approximately the same. However, at moderate rotations, the benchmark quickly stiffens and yields while the TUFF remains relatively linear throughout its range of motion, ultimately satisfying the first LEM flexure criterion. The TUFF range of motion also extends past the range of motion for the benchmark (with the TUFF undergoing a rotation nearly three times that of the benchmark before yielding), satisfying the second LEM flexure criterion. 
This section provides a generalization of the TUFF stiffness characteristics and range of motion behavior compared to that of the benchmark. This is accomplished through calculating stiffness and rotational ratios at various changes in dimensional values, while holding the material properties constant.

The vertical axes in Figures 5.7(a) and 5.7(b) contain the ratio between the angular deflection at yield for the TUFF, $\theta_{T U F F}$, over the angular deflection at yield for the benchmark,

$\theta_{\text {benchmark }}$. The higher the value of $\theta_{T U F F} / \theta_{\text {benchmark }}$, the better the performance of the TUFF over the benchmark. Figure 5.7(a) has as its horizontal the ratio of the length, $L$, over overall width, $b$, at a constant thickness, $h$. Therefore, Figure 5.7(a) provides a solution curve that shows how the difference in range of motion between the TUFF and benchmark varies as the length over width ratio varies at a constant thickness. Figure 5.7(b) is orthogonal to Figure 5.7(a), in that the horizontal is varying values of thickness at a constant the length-over-width ratio.

Figures 5.7(c) and 5.7(d) provide solution curves that show how the difference in stiffness between the TUFF, $k_{T U F F}$, and benchmark, $k_{\text {benchmark }}$, varies as $L / b$ varies at a constant $h$, or as thickness varies at a constant length-over-width ratio, respectively. Figures 5.7(c) and 5.7(d), the lower the value of $\left(k_{T U F F} / k_{\text {benchmark }}\right)$, the better the performance of the TUFF over the benchmark.

Figure 5.7 provides a basic understanding of how variations in length, width, or thickness can decease or increase the TUFF performance when compared to the LEM flexure criteria. Overall, the performance of the TUFF, in terms of range of motion $\left(\theta_{T U F F} / \theta_{\text {benchmark }}\right)$ generally decreases as both thickness and the length-over-width ratio are increased, while the performance of the TUFF, in terms of stiffness $\left(k_{T U F F} / k_{\text {benchmark }}\right)$ generally increases as both thickness and the length-over-width ratio are increased.

\subsection{TUFF Versus Benchmark: Off-axis Stiffness Ratio}

Through the stiffness characteristics analysis, it has been established that the TUFF design can successfully meet the first and second LEM flexure criterion. An off-axis stiffness ratio analysis is required to determine if the TUFF meets the off-axis stiffness ratio requirement established by the third LEM flexure criterion.

Figure 5.8(b) depicts the ideal motion for the TUFF as a rotation about the $x$-axis. The undesired axes of rotation, are the $y$ and $z$ axes. Because the length needed for the transition 


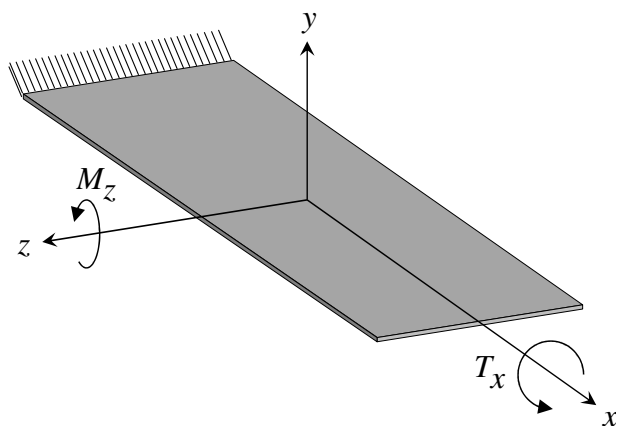

(a)

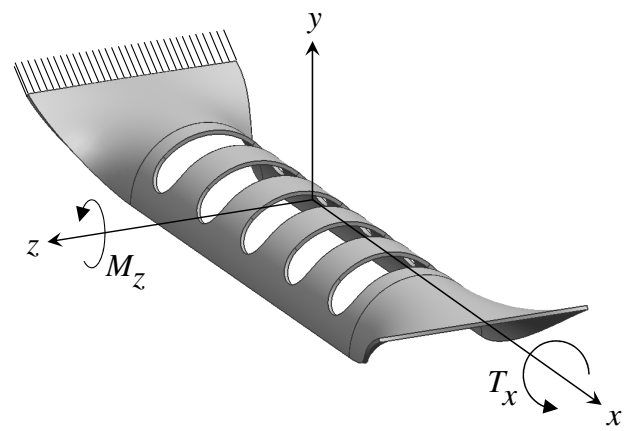

(b)

Figure 5.8: Representation of undesired $\left(M_{z}\right)$ and desired $\left(T_{x}\right)$ loading shown on both the (a) benchmark and the (b) TUFF.

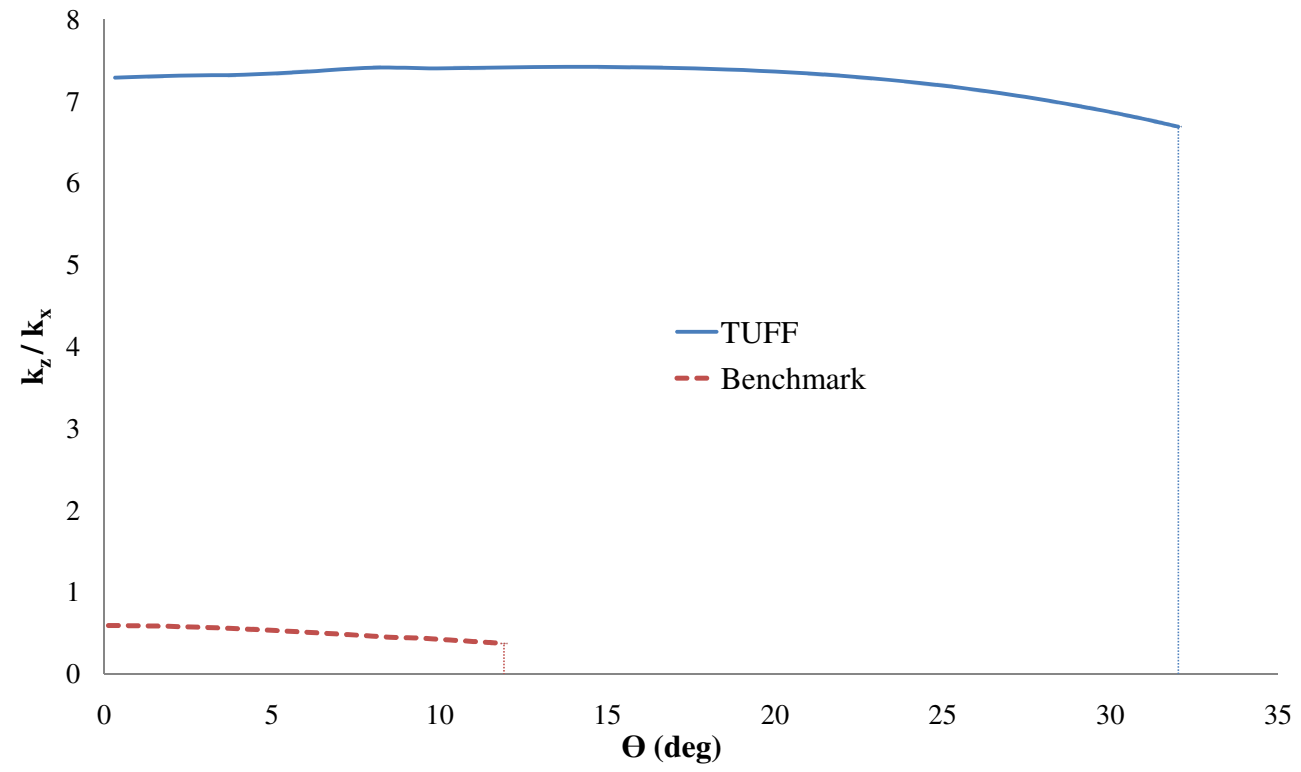

Figure 5.9: Off-axis stiffness comparison of the TUFF and benchmark.

between $\mathrm{u}$-form and straight cross-sectional profiles, the condition $L / b>1$ is usually true. The $z$-axis has been determined to be the undesired axis of most interest. To determine the robustness of the TUFF in multi-axis loading, the stiffness exhibited when subjected to a moment $M_{z}\left(k_{z}\right)$ over the stiffness exhibited when subjected to a torque $T_{x}\left(k_{x}\right)$ is compared to that of the benchmark.

An FEA model was used to calculate the off-axis stiffness ratio $k_{z} / k_{x}$. The FEA model was subjected toangular displacement, $\theta$, about both the $z$ and $x$ axes and the reaction loads $M_{z}$ and $T_{x}$ were calculated. Once the reaction loads $M_{z}$ and $T_{x}$ were known, $k_{z}$ and $k_{x}$ could be determined by 
dividing $M_{z}$ and $T_{x}$ by $\theta$, or, $M_{z} / \theta=k_{z}$ and $T_{x} / \theta=k_{x}$. Figure 5.9 provides a plot of the $k_{z} / k_{x}$ at a varying $\theta$ up to the point where the stress equals material yield strength.

The comparison of off-axis stiffness ratios for the TUFF and benchmark show that throughout the range of motion, the TUFF has an off-axis stiffness ratio approximately seven and a half times greater than the benchmark. Therefore, for the particular configuration considered, the TUFF successfully meets the third LEM flexure criterion.

\subsection{TUFF Conclusion}

The TUFF successfully meets and exceeds the LEM flexure criteria and qualifies as a viable metal LEM flexure. Thus, the TUFF provides a designer with an addition tool in the design of metal LEMs and assists in the application of LEMs to design functions, environments, and cost-effective manufacturing processes to which LEMs could previously not be easily considered. 


\section{CHAPTER 6. APPLICATION OF METAL LEM FLEXURES}

This chapter focuses on the study of an application of the RUFF and TUFF in LEMs. The study includes an analysis on how well the RUFF and TUFF can improve the maximum out-ofplane motion of a LEM over an associated benchmark LEM design. This comparison of maximum deflection will be considered as an indicator of how well the RUFF and TUFF improve upon the overall performance of a LEM.

\subsection{Compound LET Joint}

A LEM may incorporate either the RUFF or the TUFF. However, since this chapter is focused on the application of both flexures, it is ideal that they both be applied together in a single LEM. This can be accomplished through applying both the RUFF and TUFF to the basic LET joint design, and then use this new LET joint design in a LEM. Since the new LET joint design will incorporate both the RUFF and the TUFF, it will be referred to as a compound LET joint. The compound LET joint replaces the original LET joint members designed for torsion with TUFFs, and replaces the LET joint members that are designed for bending with RUFFs, as shown in Figure 6.1 (for further detail on the basic LET joint design, refer to [2]).

\subsection{RUFF and TUFF Crank-slider}

Cranks-sliders are often used as components in more complex mechanisms. As such, a LEM crank-slider has been selected for the application of the RUFF and TUFF (as a compound LET joint). The LEM crank-slider will be referred to as the RUFF and TUFF crank-slider. The maximum out-of-plane deflection of the RUFF and TUFF crank-slider will be compared to that of a benchmark crank-slider that is the same basic design as the RUFF and TUFF crank-slider, minus the RUFF and TUFF joints. 


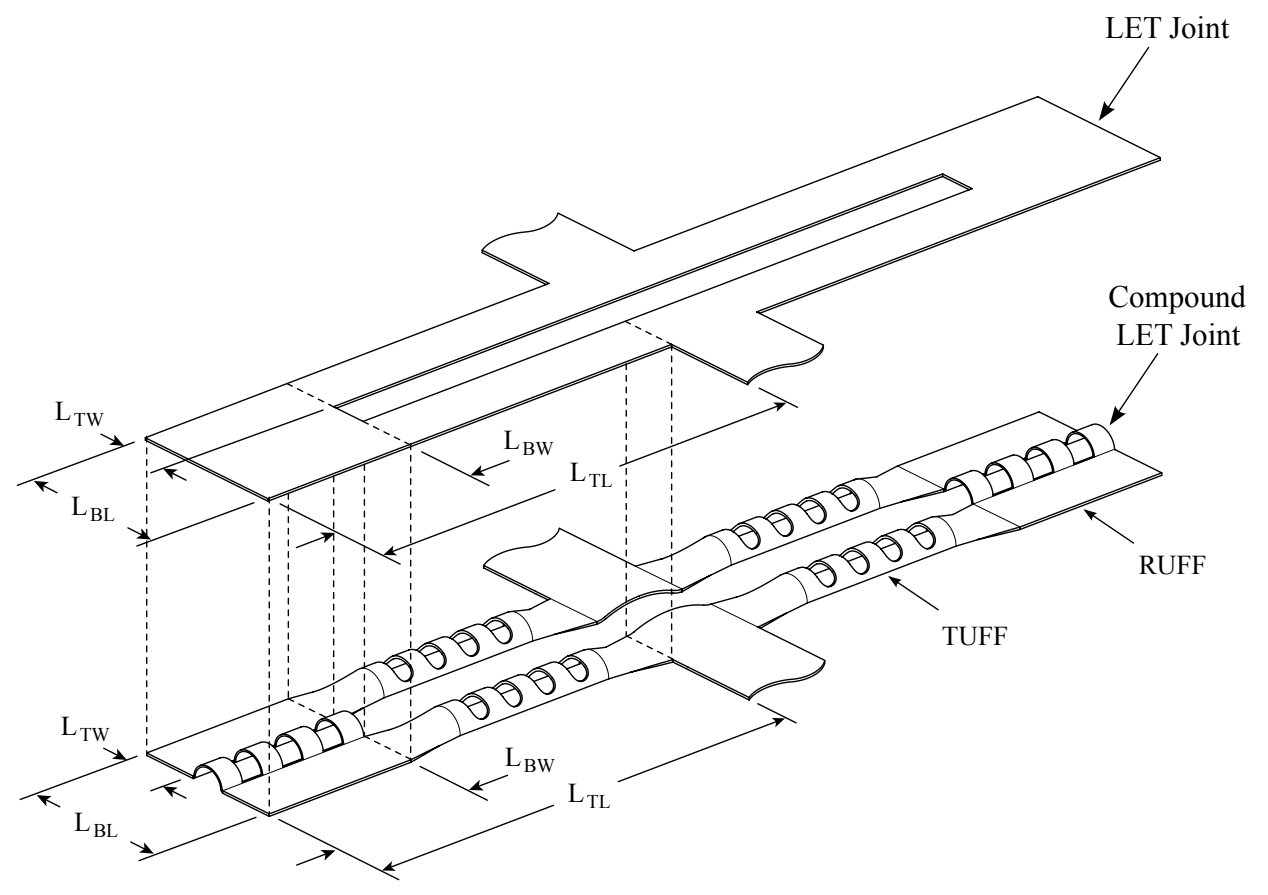

Figure 6.1: LET joint [2](top) compared to the compound LET joint (bottom), which replaces the LET members in torsion with TUFFs and the LET members in bending with RUFFs.

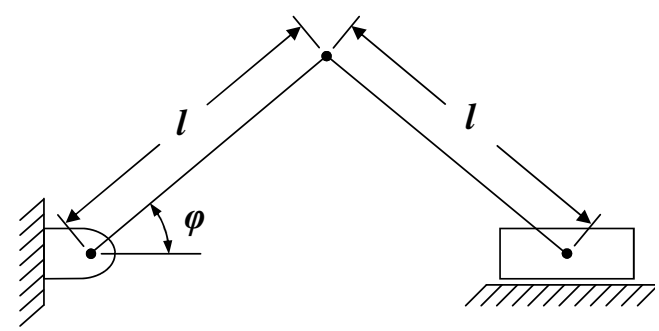

Figure 6.2: Schematic of the LEM crank-slider design.

The basic design of both crank-sliders is provided in Figure 6.2. To simplify the following deflection analysis of the RUFF and TUFF crank-slider, the members shown in Figure 6.2 are of the same length, $l$. At each of the joints shown in Figure 6.2 a compound LET joint is placed to provide the desired crank-slider motion. To provide double the amount of rotation at the middle joint over that of the end joints, the middle joint consists of two compound LET joints that are placed directly in series to one another. 


\subsection{RUFF and TUFF Crank-slider FEA and Prototype}

To analyze the maximum deflection of RUFF and TUFF crank-slider (bounded by material yield strength), an FEA model was used with the same shell elements as in the TUFF FEA analysis. The FEA model was fixed on one end, and only allowed to horizontal translation at the other end (see Figure 6.2). Since the RUFF and TUFF crank-slider is a change-point mechanism, a vertical displacement was placed directly in the center of the overall length (directly in between the two middle compound LET joints) to actuate the mechanism. The RUFF and TUFF crankslider was actuated in the middle, instead of at the end that was free to translate, so that symmetric displacement and internal stresses about both the $x$ and $z$ axes could be used to simplify the FEA model (see in Figures 6.3(a)-6.3(b)).

To illustrate the FEA-simulated physical motion of the RUFF and TUFF crank-slider a prototype was manufactured, using same processes used in the manufacture of the RUFF and TUFF prototypes. In Figures 6.3(c)-6.3(d) the RUFF and TUFF crank-slider prototype is shown in both the unactated and actuated positions.

It is important to note that the cross-section profiles of the crank-slider members were formed with a slight radius to provide stiffness to those members (see Figure 3.2) so that the majority of the deflection necessary for the RUFF and TUFF crank-slider to be actuated would be focused on each of the four joints.

An FEA model of the benchmark crank-slider was also analyzed with the same boundary conditions as the RUFF and TUFF crank-slider. The benchmark crank-slider members were also given added stiffness so that the maximum deflection of the LET joints would be more directly comparable to the maximum deflection of the compound LET joints.

\subsection{RUFF and TUFF Crank-slider FEA Results and Discussion of Results}

The results from the FEA models for both the RUFF and TUFF and benchmark cranksliders are provided in Figure 6.4. Figure 6.4 plots the increase in stress within each crank-slider, as the crank-sliders are actuated from an in-plane to a maximum out-of-plane position. This out-ofplane position is characterized by the increasing value of angle $\varphi$ (see Figure 6.2). The maximum out-of-plane position, or maximum range of motion, is bound by the yield stress of the crank- 


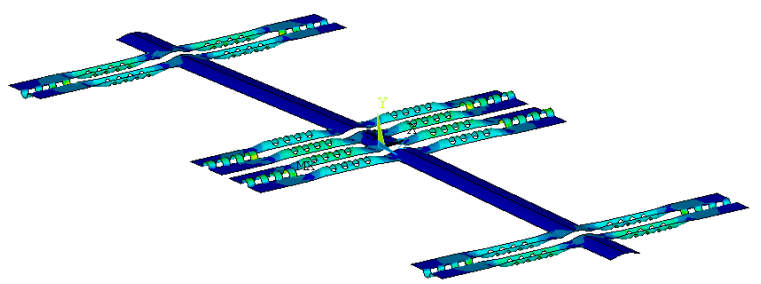

(a)

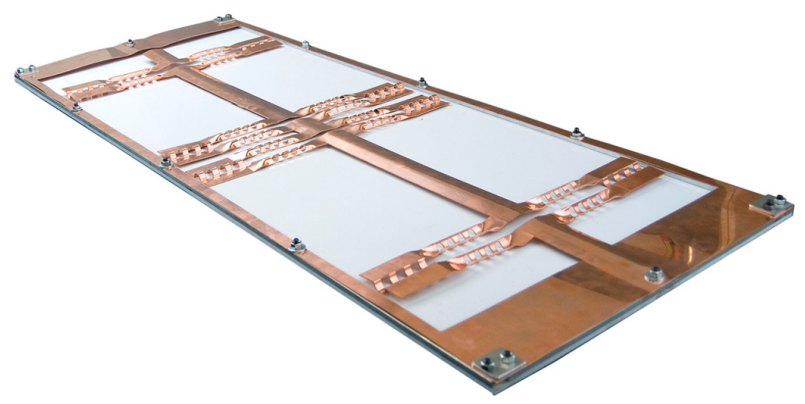

(c)

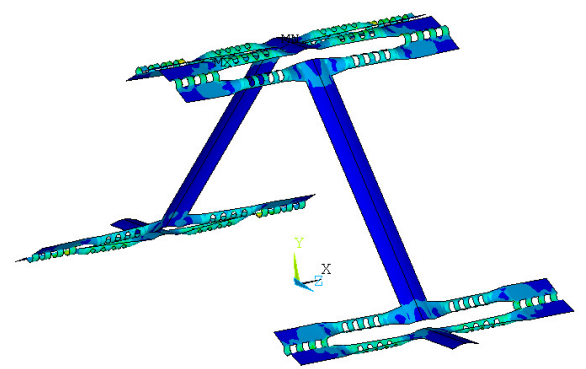

(b)

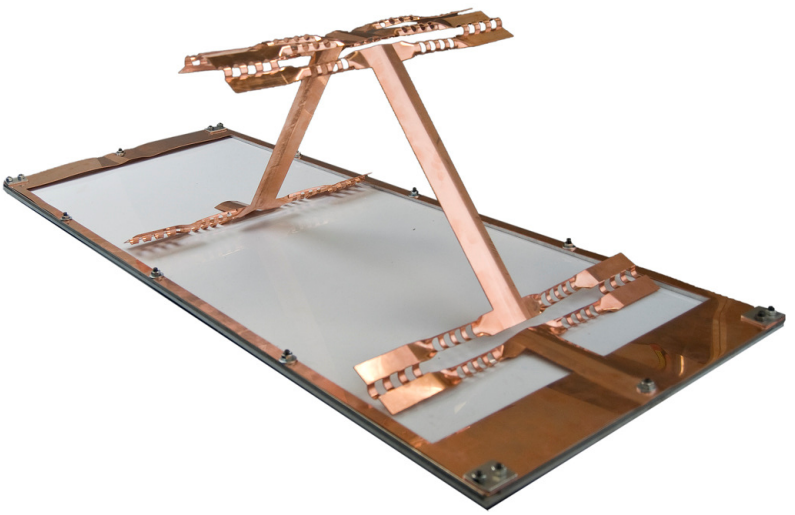

(d)

Figure 6.3: Renderings of the unactuated (a) and actuated (b) FEA model of the RUFF and TUFF crank-slider, as well as photographs of the RUFF and TUFF crank-slider prototype in both the (c) unactuated and (d) actuated positions.

slider material (24 gauge copper sheet metal was used for both the FEA model and the prototype). Thus, if the RUFF and TUFF are to provide improved performance to the crank-slider, then the range of motion of the RUFF and TUFF crank-slider should be greater than that of the benchmark crank-slider.

It should be noted that Figure 6.4 is the comparison between FEA models of both the RUFF and TUFF and benchmark crank-sliders. Force-deflection test results of the RUFF and TUFF crank-slider were not gathered. This was due to difficulties in the fabrication of the RUFFs within the crank-slider prototype with a localized decrease in thickness.

According to the FEA results shown in Figure 6.4, the RUFF and TUFF does provide a larger range of motion than that of the benchmark crank-slider. At yield, the RUFF and TUFF crank-slider shows an approximate 50\% increase in $\varphi$ over the benchmark crank-slider. 


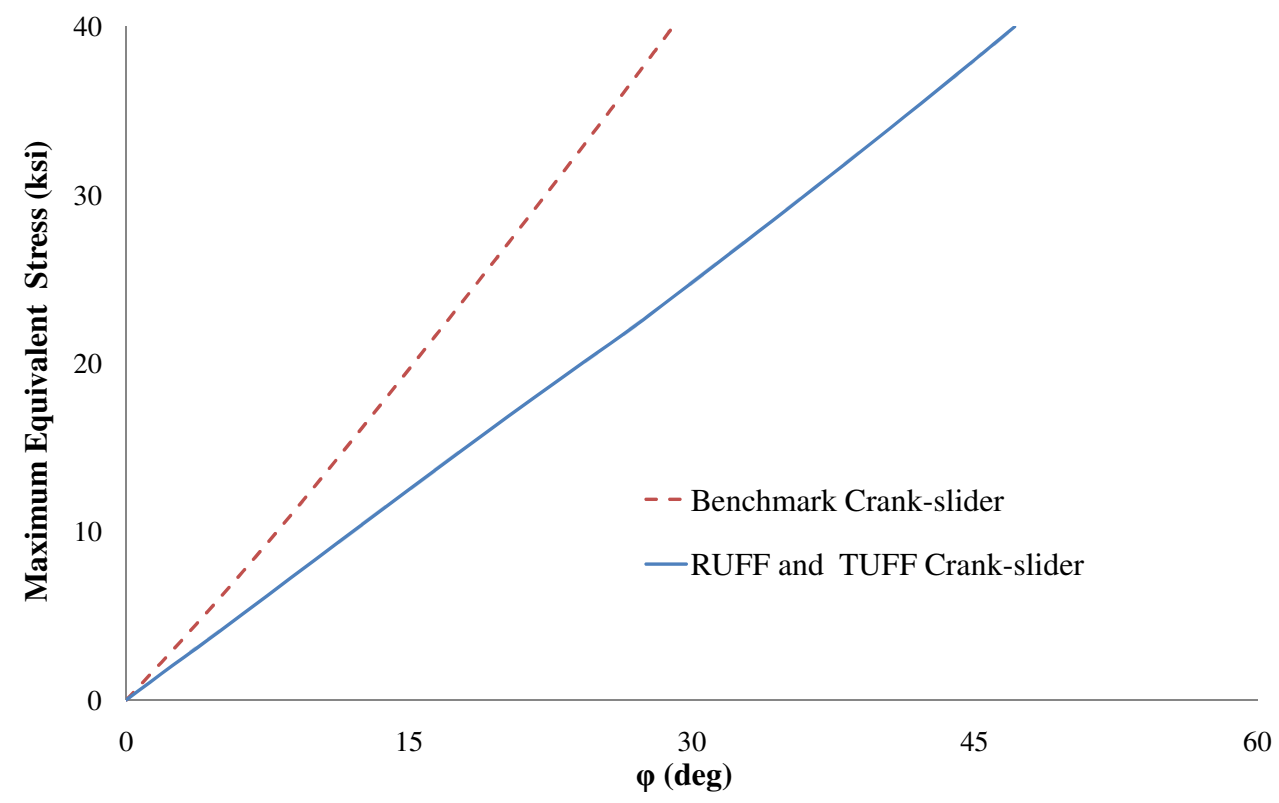

Figure 6.4: Comparision plot of the stress behavior for both the LEM crank-slider with standard LET joints and the LEM crank-slider with compound RUFF and TUFF LET joints as $\varphi$ is increased.

\subsection{Application of Metal LEM Flexures Conclusion}

It has been successfully shown that the RUFF and TUFF are able to provide improved bility. This improved maximum deflection has been shown in terms of the range of motion of a LEM, bound by the material yield strength. With the maximum deflection as an indicator of overall performance, it can be inferred that the RUFF and TUFF can provide improvements in off-axis stiffness and lower overall stiffness to a LEM.

By applying the RUFF and TUFF to a LEM, an alternative design of standard LET joint has also been introduced as the compound LET joint. Through the combination of both RUFF and TUFF, the compound LET joint shows great potential in helping to further the expansion of LEM design into sheet metals. 


\section{CHAPTER 7. CONCLUSION}

The threefold objective of this paper has been met. First, a criteria was established to qualify proposed LEM flexure designs in terms of performance relative to the benchmark. Second, the LEM flexure criteria has been successfully utilized as a tool in the design of two new LEM flexures, the RUFF and TUFF, that were developed for use in sheet metals. Along with qualifying the design of the RUFF and TUFF, the data on the mechanical characteristics of the RUFF and TUFF can be useful to a designer who is interested in using the RUFF and TUFF in a metal LEM design. Third, a successful application of the RUFF and TUFF was performed in a basic-cranks slider. In meeting the third object, a redesign of the LET join was introduced as the compound LET joint.

The discussion has introduced the idea of using the unformed, uncut, localized section of flexible LEM member as a benchmark for determining the validity of a proposed LEM flexure design by comparison of flexure performance relative to that of the benchmark. The concept of the benchmark could prove to be beneficial to future LEM research.

The potential of the LEM joint criteria to add to the LEM flexure design process has been exemplified through the design of the RUFF and TUFF. The comparative analysis process that is enabled by the criteria has been shown in the evaluation of each flexure. This potential, and the examples given by the evaluation for the RUFF and TUFF, can lead to, and be helpful in, the design of future LEM flexures for a large array of materials.

In future research, the LEM flexure criteria could be expanded to include additional criterion that would benefit the design of a LEM flexure for a particular application. For instance, in situations when a LEM flexure will need to be designed to a specific fatigue limit, the current LEM flexure criteria could be expanded to include a specification on how a LEM flexure should improve on the fatigue life of the benchmark. In this research the LEM flexure criteria has been used to design flexures with one degree of freedom, however the potential is there to design flexures with 
multiple degrees of freedom. However, perhaps a focusing, or modification, of the LEM flexure criteria would be helpful in the design of multiple-degree-of-freedom flexures.

For future research in metal LEM flexures; the changes in the metal materials induced by manufacturing process used to cut and form the flexures, and the effects those changes have on the mechanical behavior of the LEM flexures, could be explored. Specifically, future research could include the effects of work hardening on the overall performance of a LEM flexure. Additionally, in expanding metal LEM research, the advantages and disadvantages of using different types of metals, other than copper, could be examined for specific applications. Exploration of the effects of using different types of metals could be especially useful in multi-layered metal LEM applications. 


\section{REFERENCES}

[1] Parise, J. J., Howell, L. L., and Magleby, S. P., 2001. "Ortho-planar linear-motion springs." Mechanism and Machine Theory, 36, pp. 1281-1299.

[2] Jacobsen, J. O., Chen, G., Howell, L. L., and Magleby, S. P., 2009. "Lamina emergent torsional (LET) joint." Mechanism and Machine Theory, 44, pp. 2098-2109.

[3] Howell, L. L., and Midha, A., 1994. "A method for the design of compliant mechanisms with small-length flexural pivots." Journal of Mechanical Design, Transactions of the ASME, 116, pp. 280-290.

[4] Norton, R. L., 2008. Design of Machinery. McGraw Hill.

[5] Lusk, C. P., and Howell, L. L., 2006. "Design space of single-loop planar folded micro mechanisms with out-of-plane motion." Journal of Mechanical Design, Transactions of the ASME, 128, pp. 1092-1100.

[6] Jacobsen, J. O., Winder, B. G., Howell, L. L., and Magleby, S. P., 2010. "Lamina emergent mechanisms and their basic elements." Journal of Mechanisms and Robotics, 2, pp. 011003 (1-9).

[7] Winder, B. G., Magleby, S. P., and Howell, L. L., 2008. "A study of joints suitable for lamina emergent mechanisms." In 2008 Proceedings of the ASME International Design Engineering Technical Conferences and Computers and Information in Engineering Conference.

[8] Howell, L. L., 2001. Compliant Mechanisms. Wiley-Interscience.

[9] Goldfarb, M., and Speich, J. E., 1999. "A well-behaved revolute flexure joint for compliant mechanism design." Journal of Mechanical Design, Transactions of the ASME, 121, pp. 424429.

[10] Dai, J. S., and Jones, J. R., 1999. "Mobility in metamorphic mechanisms of foldable/erectable kinds." Journal of Mechanical Design, Transactions of the ASME, 121, pp. 375-382.

[11] Frecker, M. I., Ananthasuresh, G. K., Nishiwaki, S., Kikuchi, N., and Kota, S., 1997. "Topological sythesis of compliant mechanisms using multi-criteria optimization." Journal of Mechanical Design, Transactions of the ASME, 119, pp. 238-245.

[12] Jang, G.-W., Kim, M.-J., and Kim, Y. Y., 2009. "Design optimization of compliant mechanisms consisting of standardized elements." Journal of Mechanical Design, Transactions of the ASME, 131, p. 121006 (8 pages).

[13] Trease, B. P., Moon, Y.-M., and Kota, S., 2005. "Design of large-displacement compliant joints." Journal of Mechanical Design, Transactions of the ASME, 127, pp. 788-798. 
[14] Deepak, S. R., 2008. "A global constraint on relative rotation to avoid lumped compliant mechanisms in topology optimization." In 2008 Proceedings of the ASME International Design Engineering Technical Conferences and Computers and Information in Engineering Conference.

[15] Qiu, L., Weng, H., Liu, L., and Nan, T., 2007. "Study on complete compliant mechanisms of incompletion distributed compliance." Journal of University of Sicence and Technology, 29, pp. 156-158.

[16] Guerinot, A. E., Magleby, S. P., Howell, L. L., and Todd, R. H., 2005. "Compliant joint design principles for high compressive load situations." Journal of Mechanical Design, Transactions of the ASME, 127, pp. 774-781.

[17] Norton, R. L., 2006. Machine Design. Pearson Prentice Hall.

[18] Jacobsen, J. O., Howell, L. L., and Magleby, S. P., 2008. "Components for the design of lamina emergent mechanisms." In ASME International Mechanical Engineering Congress and Exposition, Proceedings.

[19] Dai, J. S., and Cannella, F., 2008. "Stiffness characteristics of carton folds for packaging." Journal of Mechanical Design, Transactions of the ASME, 130, p. 022305.

[20] Budynas, R. G., and Nisbett, J. K., 2008. Shigley’s Mechanical Engineering Design. McGraw Hill. 